\title{
Multi-level meta-analysis of single-case experimental designs using robust variance estimation
}

\author{
Man Chen $^{1} \&$ James E. Pustejovsky ${ }^{1}$ \\ ${ }^{1}$ University of Wisconsin-Madison
}

(C2022, American Psychological Association. This paper is not the version of record and may not exactly replicate the final, published version of the article. The final article will be available, upon publication, via its DOI: 10.1037/met0000510

\begin{abstract}
Single-case experimental designs (SCEDs) are used to study the effects of interventions on the behavior of individual cases, by making comparisons between repeated measurements of an outcome under different conditions. In research areas where SCEDs are prevalent, there is a need for methods to synthesize results across multiple studies. One approach to synthesis uses a multi-level meta-analysis (MLMA) model to describe the distribution of effect sizes across studies and across cases within studies. However, MLMA relies on having accurate sampling variances of effect size estimates for each case, which may not be possible due to auto-correlation in the raw data series. One possible solution is to combine MLMA with robust variance estimation (RVE), which provides valid assessments of uncertainty even if the sampling variances of effect size estimates are inaccurate. Another possible solution is to forgo MLMA and use simpler, ordinary least squares (OLS) methods with RVE. This study evaluates the performance of effect size estimators and methods of synthesizing SCEDs in the presence of auto-correlation, for several different effect size metrics, via a Monte Carlo simulation designed to emulate the features of real data series. Results demonstrate that the MLMA model with RVE performs properly in terms of bias, accuracy, and confidence interval coverage for estimating overall average log response ratios. The OLS estimator corrected with RVE performs the best in estimating overall average Tau effect sizes. None of the available methods perform adequately for meta-analysis of within-case standardized mean differences.
\end{abstract}

Keywords: meta-analysis; single-case experimental designs; log response ratio; non-overlap of all pairs; within-case standardized mean difference

Single-case experimental designs (SCEDs) are a set of methods for studying the effects of interventions on the behavior of individual cases. Cases may correspond to individual persons, to groups of individuals in a common context, or even to a single individual observed across multiple settings. The central feature of all SCEDs is repeated measurement of a behavior under different treatment conditions (e.g., presence or absence of an intervention), which are often structured into temporal phases, such as an initial baseline phase followed by a treatment phase. These features allow intervention effects to be identified by making comparisons of outcomes across phases or conditions within each case (Horner \& Odom, 2014).

SCEDs have been employed in a variety of disciplines within education and psychology, such as behavior analysis, clinical psychology, and school psychology (Kratochwill \& Levin, 2014). SCEDs can be applied with a small number of participants, or even a single partic- ipant, making them an attractive tool for studying interventions for individuals with low-incidence disabilities and other populations where it can be difficult to assemble a sufficiently large and homogeneous sample to conduct a between-groups experimental design. Furthermore, SCEDs can be implemented with interventions that are adapted or tailored for individual participants and their contexts, allowing for continual refinement of intervention strategies (Kaiser, 2014).

Although a given SCED study can provide valuable, clinically relevant evidence about the effects of an intervention for the individual participants, it is difficult to gauge the extent to which results from a single study generalize to a broader population. However, in some areas of research where SCEDs are widely applied, results are available from multiple studies investigating a conceptually similar intervention. Meta-analysis methods may be used to synthesize results across multiple studies and draw more generalized, externally valid conclusions 
about the overall effects of intervention, about variability in effects, and about factors associated with larger or smaller effects (Pustejovsky \& Ferron, 2017). For example, Collins et al. (2020) synthesized findings from 21 single-case research studies to evaluate the effects of peer reporting interventions on student outcomes, including disruptive behavior, academically engaged behavior, and social behavior. Among other moderator analyses, the authors found that effect sizes from published studies were significantly larger than those from unpublished studies.

Two broad approaches for synthesizing SCED data have been proposed: a one-stage approach and a two-stage approach (Declercq et al., 2020; Pustejovsky \& Ferron, 2017; Van den Noortgate \& Onghena, 2008). Both approaches involve extracting raw outcome data and design information from graphs, which are typically reported in the primary studies. In the one-stage approach, one synthesizes the raw data from multiple SCED studies using a hierarchical linear model that describes variation in the outcomes within cases, across cases, and across studies (Van den Noortgate \& Onghena, 2008). This approach is relevant when all studies use identical methods of assessing the outcomes, or when the outcomes can be standardized or equated in some way.

In the second, two-stage approach, one first calculates effect size estimates, each of which describe the intervention effect for a single case within a single study. This results in a hierarchical structure where case-level effect size estimates are nested within studies. One then synthesizes the effect size estimates with a multi-level meta-analytic (MLMA) model, yielding an overall average intervention effect and descriptions of heterogeneity

Man Chen, Department of Educational Psychology, University of Wisconsin-Madison. James E. Pustejovsky, Department of Educational Psychology, University of Wisconsin-Madison.

This work was supported by Grant R324U190002 from the Institute of Educational Sciences, U.S. Department of Education. The opinions expressed are those of the authors and do not represent the views of the Institute or the U.S. Department of Education.

An earlier version of this paper was presented at the annual convention of the American Educational Research Association, April 12, 2021 online.

A preprint of the manuscript is available at https://psyarxiv. com $/ 59 \mathrm{~h} 32$. The code for replicating the simulations and complete numerical simulation results are available on the Open Science Framework at https://osf.io/zypsh.

Correspondence concerning this article should be addressed to Man Chen, 1025 W Johnson St, Madison, WI 53706. E-mail: mchen277@wisc.edu in effect sizes at the case level and study level (Pustejovsky \& Ferron, 2017; Van den Noortgate \& Onghena, 2008). The MLMA model can also be extended to include a meta-regression for examining associations between effect size and case-level or study-level characteristics. The two-stage approach is relevant when studies use different methods of assessing outcomes-making one-stage approaches infeasible-but where effect size metrics can be calculated and compared across studies. In the present study, we will focus on the two-stage approach because it is comparatively simpler and has been more widely applied (Jamshidi et al., 2020).

The two-stage approach to synthesis requires choosing an effect size metric that quantifies the direction and magnitude of an intervention effect for each case within an SCED. Among the available effect size measures, the log response ratio (LRR, Pustejovsky, 2018), withincase standardized mean difference (WC-SMD, Busk \& Serlin, 1992; Gingerich, 1984), non-overlap of all pairs (NAP, Parker \& Vannest, 2009), and Tau index (Parker, Vannest, Davis, \& Sauber, 2011) are suitable for metaanalysis because they have known sampling variances. However, available formulas for the sampling variances of these indices are all based on the assumption that outcome measurements are independent within each measurement phase. This assumption would be violated if outcomes are instead auto-correlated.

Because SCEDs involve repeated measurements across time on a single unit, it is plausible that the data series for each case could exhibit auto-correlation (Horner, Swaminathan, Sugai, \& Smolkowski, 2012; Matyas \& Greenwood, 1996). In the presence of positive autocorrelation, the sampling variances of the effect size estimates will be downwardly biased, which could lead to inflated Type I error rates and inaccurate statistical inferences about the intervention effects (Petit-Bois, Baek, Van den Noortgate, Beretvas, \& Ferron, 2016). However, the implications of auto-correlation for multi-level metaanalysis of these effect sizes has yet to be examined.

In meta-analysis of between-groups experimental designs, robust variance estimation methods (RVE) have been widely used for drawing inferences regarding overall average intervention effect estimates and metaregression coefficients (Hedges, Tipton, \& Johnson, 2010; Tipton, 2015). Pustejovsky (2018) proposed the use of RVE in conjunction with multi-level meta-analysis of SCEDs using LRR effect sizes, arguing that RVE can provide valid assessments of uncertainty even if the sampling variances of effect sizes might be inaccurate due to auto-correlation. Pustejovsky (2018) illustrated the approach in an empirical application. The approach has also been applied in several recent syntheses of SCEDs (e.g., Collins et al., 2020; Froján-Parga, Prado- 
Gordillo, Álvarez-Iglesias, \& Alonso-Vega, 2019; Ledford \& Pustejovsky, 2020; Wahman, Pustejovsky, Ostrosky, \& Santos, 2019; Zimmerman et al., 2018).

Despite increasing interest and application, no methodological research has assessed the performance of MLMA and RVE for synthesizing single-case data. In this study, we used simulation methods to evaluate the performance of this combination of methods, focusing specifically on its performance when applied to autocorrelated data series, where conventional standard errors for effect size estimators would be expected to perform poorly. Further, we examine the approach for several different effect size metrics for SCEDs, including the LRR, WC-SMD, and Tau index. Finally, we compare the MLMA model to the simpler alternative of ordinary least squares (OLS) estimation, combined with RVE. We designed simulations that emulate several key features of real data from SCEDs, so that findings are relevant to the practical application of SCED meta-analysis.

The remainder of the paper is structured as follows. In the next section, we review several within-case effect size metrics and their statistical properties. In the following section, we describe the issue of auto-correlation in SCED data and investigate the implications of autocorrelation for the bias and standard error of these effect sizes. We then describe the MLMA and OLS approaches to meta-analysis of SCED effect sizes, along with the use of RVE in conjunction with these two methods. The subsequent two sections report the methods and results from a simulation study to assess the performance of those estimators, including bias, accuracy, and confidence interval coverage. The final section concludes with a discussion of findings, limitations, directions for future research, and implications for practice.

\section{Effect sizes for SCEDs}

A wide variety of effect size measures have been proposed for use specifically with SCEDs (see Manolov \& Moeyaert, 2017; Parker, Vannest, \& Davis, 2011; Pustejovsky, 2019 for expansive reviews). In this section, we describe a selected set of these effect size indices, including LRR, WC-SMD, NAP, and Tau index. We focus on these particular indices for several reasons. First, all four indices quantify the direction and magnitude of effects at the level of the case and thus provide a means to study heterogeneity of intervention effects across individuals. Second, all four indices have stable parameter definitions that are functions of the marginal distribution of outcomes under the baseline and intervention conditions. ${ }^{1}$ Finally, all four indices are appropriate for data that do not exhibit time trends within the baseline phase or intervention phase. Although accounting for time trends is clearly important in some applications, modeling time trends introduces additional complexity that is orthogonal to understanding the consequences of autocorrelation. Furthermore, effect size indices that assume stable baseline and intervention phases have been widely used in meta-analysis of SCEDs (Maggin, O'Keeffe, \& Johnson, 2011), indicating that this is an important scenario to consider.

The effect size indices under consideration can be defined based on a simple model for the data for a pair of phases within a SCED. Suppose that the baseline phase consists of $m$ measurements, $y_{1}, \ldots, y_{m}$, and that the subsequent intervention phase consists of $n$ measurements, $y_{m+1}, \ldots, y_{m+n}$. For developing the sampling properties of the effect size indices, we shall assume that the outcome measurements within the baseline phase and the intervention phase are independent realizations of the random variables $Y^{A}$ and $Y^{B}$, respectively. Let $\mu_{A}$ and $\sigma_{A}^{2}$ denote the expectation and variance of $Y^{A}$; similarly, let $\mu_{B}$ and $\sigma_{B}^{2}$ denote the expectation and variance of $Y^{B}$. Although we assume that the measurements are independent here, the simulations will examine the performance of the effect size indices when this assumption is violated.

\section{Log response ratio}

The log response ratio (LRR) is an effect size that quantifies treatment effect magnitude in terms of proportionate change in the level of the outcome from the baseline phase to the treatment phase. Recalling that $\mu_{A}$ and $\mu_{B}$ denote the mean outcomes in the baseline and treatment phases respectively, the LRR parameter is defined as

$$
\lambda=\ln \left(\mu_{B} / \mu_{A}\right)
$$

where $\ln ()$ denotes the natural logarithm function. The natural logarithm is applied because it makes the range of the effect size less restricted. If intervention has no effect, so that $\mu_{B}=\mu_{A}$, then $\lambda=0$.

Pustejovsky (2015) introduced the LRR as an effect size for SCEDs, arguing that it is a particularly useful metric for the types of behavioral measurements that are commonly used as outcomes in SCEDs. Such behavioral outcome measures are often in the form of frequency counts or proportions of time, for which ratio comparisons are

\footnotetext{
${ }^{1}$ There are several other well known and widely applied non-overlap indices, including the percentage of nonoverlapping data, the percentage of all non-overlapping data, and the robust improvement rate difference (Parker, Vannest, \& Davis, 2011). However, these indices are influenced by the number of observations during the baseline or intervention phase, which makes it difficult to interpret them as measures of effect magnitude (Pustejovsky, 2019).
} 
meaningful. For example, Wahman et al. (2019) conducted a meta-analysis of SCEDs to examine the effectiveness of social stories interventions on the behaviors of young children, including challenging behavior and pro-social skills. The authors used LRR to quantify treatment effects for each type of behavior.

Pustejovsky (2015) described two estimators of the LRR: a basic moment estimator and a bias-corrected estimator. The basic moment estimator is constructed by replacing the mean parameters with corresponding sample means:

$$
L R R_{1}=\ln \left(\bar{y}_{B} / \bar{y}_{A}\right)=\ln \left(\bar{y}_{B}\right)-\ln \left(\bar{y}_{A}\right) .
$$

However, this estimator is biased when either phase includes only a small number of observations. A biascorrected estimator is given by

$$
L R R_{2}=\ln \left(\bar{y}_{B}\right)+\frac{s_{B}^{2}}{2 n_{B} \bar{y}_{B}^{2}}-\ln \left(\bar{y}_{A}\right)-\frac{s_{A}^{2}}{2 n_{A} \bar{y}_{A}^{2}} .
$$

Again assuming that the outcome measurements are mutually independent, an approximate estimate of the sampling variance of either estimator is

$$
V^{L R R}=\frac{s_{A}^{2}}{n_{A} \bar{y}_{A}^{2}}+\frac{s_{B}^{2}}{n_{B} \bar{y}_{B}^{2}} .
$$

The simulations in subsequent sections examine the performance of both $L R R_{1}$ and $L R R_{2}$. In the simulations and in practice, we use truncated sample means and sample variances (Pustejovsky, 2018) to handle the possibility that the sample means might be zero. Using these truncated statistics is necessary to ensure that the effect size estimators are always finite and that the variance estimator is well defined and strictly positive.

\section{Within-case standardized mean difference}

The standardized mean difference is one of the most widely used effect size indices in meta-analysis of between-groups studies, and methods for estimating it are available for a range of experimental designs (Borenstein, 2009). Arguing by analogy to its use in betweengroups designs, Gingerich (1984) proposed a version of the metric for quantifying effect size in SCEDs, but defined in terms of within-case variability in the outcome (see also Busk \& Serlin, 1992). This within-case standardized mean difference (WC-SMD) effect size is

$$
\delta=\frac{\mu_{B}-\mu_{A}}{\sigma_{A}} .
$$

This parameter is conceptually distinct from-and not directly comparable to-the standardized mean difference parameter for a between-groups experimental design (Shadish, Hedges, \& Pustejovsky, 2014; Van den
Noortgate \& Onghena, 2008). This is because $\sigma_{A}$ captures the standard deviation of the outcome measure under the baseline condition for an individual case, whereas the denominator for a between-groups design includes both within- and between-individual variability.

In a methodological review of previous meta-analyses that included SCDs, Maggin, O'Keeffe, et al. (2011) found that the WC-SMD is one of the more frequently applied effect size indices, second only to the percentage of non-overlapping data. For example, Randolph (2007) used the WC-SMD to synthesize results from SCEDs on the effects of using response cards to improve academic achievement. The WC-SMD also provides the conceptual basis of standardization procedures used in conjunction with recently developed one-stage approaches to synthesis of SCEDs (Moeyaert, Ugille, Ferron, Beretvas, \& Van den Noortgate, 2013).

Let $\bar{y}_{A}$ and $\bar{y}_{B}$ denote the sample means and $s_{A}$ and $s_{B}$ denote the sample standard deviations of the outcomes in the baseline and intervention phases, respectively. Gingerich (1984) proposed to estimate $\delta$ using

$$
d=\frac{\bar{y}_{B}-\bar{y}_{A}}{s_{A}} \text {. }
$$

However, this estimator has a non-negligible bias if the baseline phase consists of only a few measurements. A bias-corrected estimator of $\delta$ is given by

$$
g=\left(1-\frac{3}{4 m-5}\right)\left(\frac{\bar{y}_{B}-\bar{y}_{A}}{s_{A}}\right)
$$

(Hedges, 1981; Ugille, Moeyaert, Beretvas, Ferron, \& Van den Noortgate, 2014). Under the assumption that the outcome measurements are mutually independent, the sampling variance of $g$ can be estimated using

$$
V^{g}=\left(1-\frac{3}{4 m-5}\right)^{2}\left(\frac{1}{m}+\frac{s_{B}^{2}}{n s_{A}^{2}}+\frac{d^{2}}{2(m-1)}\right),
$$

which is a variant of the conventional formula (Borenstein, 2009), allowing for the possibility that the variance of the outcome during the baseline phase may differ from the variance during the treatment phase. In practice and in the subsequent simulation study, we truncate sample standard deviations of zero at the value of $s_{A}=m^{-3 / 2}$ and $s_{B}=n^{-3 / 2}$ to ensure that $g$ remains well-defined and that $V^{g}$ is strictly positive.

\section{Non-overlap of all pairs}

NAP (Parker \& Vannest, 2009) is an effect size index in the family of non-overlap measures, calculated by comparing each observation in the intervention phase to each 
observation in the baseline phase. NAP is defined as the proportion of all pair-wise comparisons where the intervention phase observation represents a therapeutic improvement over the baseline phase observation, with tied observations counted as 0.5 . Let $q_{i j}=1$ if $y_{m+j}$ is an improvement over $y_{i}$ (i.e., $y_{m+j}>y_{i}$ if increasing the outcome is desirable; $y_{m+j}<y_{i}$ if decreasing the outcome is desirable); let $q_{i j}=0.5$ if $y_{j}^{B}=y_{i}^{A}$; and let $q_{i j}=0$ otherwise, for $i=1, \ldots, m$ and $j=1, \ldots, n$. NAP is then calculated as

$$
\mathrm{NAP}=\frac{1}{m n} \sum_{i=1}^{m} \sum_{j=1}^{n} q_{i j}
$$

The NAP statistic can be interpreted as an estimate of a stable effect size parameter measuring the degree of overlap between the distributions of $Y^{A}$ and $Y^{B}$. The definition of the parameter depends on the direction of therapeutic benefit for the outcome, with

$$
\begin{aligned}
\theta=0.5 \times \operatorname{Pr}\left(Y^{B}=Y^{A}\right) \\
+ \begin{cases}\operatorname{Pr}\left(Y^{B}>Y^{A}\right) & \text { if increase is desirable } \\
\operatorname{Pr}\left(Y^{B}<Y^{A}\right) & \text { if decrease is desirable }\end{cases}
\end{aligned}
$$

Values of $\theta=0.5$ correspond to no effect of treatment, while $\theta>0.5$ indicates therapeutic improvement.

Parker \& Vannest (2009) argued that NAP has advantages over other non-overlap indices used with SCEDs because it makes use of all pairs of the measurements, making it more stable and less sensitive to outliers than indices such as the percentage of non-overlapping data. Furthermore, indices equivalent to NAP have been proposed as effect sizes in other research contexts (e.g., Acion, Peterson, Temple, \& Arndt, 2006). NAP effect sizes have been used in practice to synthesize results across multiple SCEDs. For instance, Bene, Banda, \& Brown (2014) conducted a meta-analysis of NAP effect sizes to synthesize results from SCEDs on the effects of peer-mediated instructional arrangements for children with autism spectrum disorders.

Because of the correspondence between NAP and effect size indices used in other disciplines, previous statistical research has considered how to quantify the uncertainty of NAP as an estimate of $\theta$. Sen (1967) described several methods for estimating the sampling variance of an index that is equivalent to NAP, assuming that the outcome measurements are mutually independent. An unbiased estimator of the variance of NAP is given by

$$
V^{N A P}=\frac{\mathrm{NAP} \times(1-\mathrm{NAP})+n Q_{1}+m Q_{2}-2 Q_{3}}{(m-1)(n-1)},
$$

where

$$
\begin{aligned}
& Q_{1}=\frac{1}{m n^{2}} \sum_{i=1}^{m}\left(\sum_{j=1}^{n}\left(q_{i j}-\mathrm{NAP}\right)\right)^{2} \\
& Q_{2}=\frac{1}{m^{2} n} \sum_{j=1}^{n}\left(\sum_{i=1}^{m}\left(q_{i j}-\mathrm{NAP}\right)\right)^{2} \\
& Q_{3}=\frac{1}{m n} \sum_{i=1}^{m} \sum_{j=1}^{n}\left(q_{i j}-\mathrm{NAP}\right)^{2}
\end{aligned}
$$

(Sen, 1967; see also Mee, 1990). Although unbiased, this variance estimator also has the property that it is equal to zero when NAP is equal to zero or one (i.e., when there is no overlap between phases). To ensure that the variance is strictly positive, we will consider calculating $V^{N A P}$ by replacing NAP with the value of $\frac{1}{2 m n}$ if $\mathrm{NAP}=0$ and with $\frac{2 m n-1}{2 m n}$ if $\mathrm{NAP}=1$.

Tau

Parker, Vannest, Davis, \& Sauber (2011) proposed the Tau effect size for SCEDs, as the basis for several nonoverlap measures that include modifications for time trends in the baseline and treatment phases. Without any adjustments for time trends, Tau is a simple linear transformation of NAP (Pustejovsky, 2019):

$$
\mathrm{Tau}=2 \times \mathrm{NAP}-1 .
$$

An estimator for the sampling variance of Tau can therefore be calculated as

$$
V^{T a u}=4 \times V^{N A P} .
$$

Tau ranges from -1 to 1 , with -1 corresponding to complete non-overlap in the undesirable direction and 1 corresponding to complete non-overlap in the desirable direction. Zero corresponds to no effect, so that the distributions of $Y^{A}$ and $Y^{B}$ are stochastically equal. Because Tau is a simple linear transformation of NAP, the performance of meta-analysis methods based on Tau are equally relevant for meta-analysis of NAP. In the simulations, we focused on Tau rather than NAP because its scale and null value are more similar to the other, parametric measures that we have described.

\section{Auto-correlation in SCEDs}

The features of SCED data present several challenges for statistical analysis and meta-analysis (Horner et al., 2012; Maggin, Swaminathan, et al., 2011; Wolery, Busick, Reichow, \& Barton, 2010). Outcomes in SCEDs are often assessed using systematic direct observation 
method, leading to measurements that are non-normally distributed (Pustejovsky, Swan, \& English, 2019). Because the outcome measurements are collected repeatedly over time for each case, models may need to account for features such as systematic time trends or changes in level or variability over time. Further, it is possible that measurements that are closer in time will tend to be more similar to each other to measurements from more distant time points, indicating auto-correlation in the within-case errors. These features are all challenging to assess and model because SCED data typically include a very limited number of observations in each phase or condition. In the present study, we focus on the challenges presented by auto-correlation, leaving aside questions of modeling time trends or shifts in level or variability, in order to thoroughly understand its consequences and evaluate potential analytic solutions.

Auto-correlation presents challenges for statistical analyses that rely on an assumption that measurements are independent (Horner et al., 2012; Solomon, 2014). Previous research has found evidence of varying levels of auto-correlation in SCEDs. Shadish \& Sullivan (2011) examined the characteristics of 113 single-case studies in various fields of education and psychology. They estimated an average lag-one auto-correlation of 0.2 $(\mathrm{SE}=0.018)$, significantly different than zero. They also found that auto-correlation varied across different types of single-case designs. For example, the average auto-correlation was 0.32 for multiple baseline designs but 0.19 for reversal designs. In an evaluation of violations of normality and independence assumptions in SCED data, Solomon (2014) estimated levels of auto-correlation across several collections of studies from different domains of school-based SCED research. He found an average lag-one auto-correlation of $0.06,95 \%$ CI $[-.01, .13]$, but with a substantial degree of heterogeneity within and across domains. BarnardBrak, Watkins, \& Richman (2021) estimated the autocorrelation of 304 data series from two meta-analyses of single-case studies and obtained an overall average autocorrelation of 0.46 with a standard deviation of 0.33 .

Some previous research has investigated the consequences of auto-correlation for the one-stage (raw data) approach to meta-analysis of SCEDs (Petit-Bois et al., 2016). However, the implications of auto-correlation for the two-stage approach, based on within-case effect size measures, warrants further investigation. There are several reasons that auto-correlation may present problems. First, the sampling variances of LRR, WC-SMD, NAP and Tau index described in the previous section are based on the assumption that the outcome measurements are mutually independent. When the outcome data are positively correlated within each case, we expect that the sampling variances of the those effect size estimates will be under-estimated. Using under-estimated sampling variances in the MLMA model might result in inaccurate estimates of the variance components and standard errors for the overall average effect size, leading to inappropriate statistical inference about the intervention effects.

A second possibility is that the effect size estimators themselves could be biased. In particular, the bias corrections used in estimating the LRR and WC-SMD are developed under a model with independent observations (Hedges, 1981; Pustejovsky, 2015), and so it is possible that they do not work properly in the presence of auto-correlation. Moreover, the WC-SMD effect size estimator uses the sample standard deviation $s_{A}$ to estimate the population standard deviation of the outcomes in the baseline phase $\sigma_{A}$. It is expected that $s_{A}$ will be underestimated if the baseline data series demonstrate positive auto-correlation, especially for short phase lengths (Anderson, 1994, p. 448). As a result, the WC-SMD effect size estimator may be upwardly biased. ${ }^{2}$ Generally, using biased effect size estimators in the MLMA model could lead to systematically biased estimates of average effects and, consequently, inappropriate inferences.

\section{Effect size simulations}

Before considering the implications of auto-correlation for meta-analysis of within-case effect size estimators, it is useful to understand the properties of the effect size estimators taken singly. We therefore conducted simulations to examine the properties of the LRR, WC-SMD, and Tau effect size indices and their corresponding variance estimators when applied to auto-correlated data series. Previous methodological research on SCED effect size indices has focused largely on data-generating models with normally distributed (i.e., Gaussian) errors (Moeyaert et al., 2013; Petit-Bois et al., 2016). However, outcome measures in SCEDs are often in the form of frequency counts or proportions, which may be poorly approximated by normal distributions. As a potentially more realistic data-generating model for frequency count outcomes, we use a serially correlated Poisson model for simulating raw data. (We present parallel results based on a model with serially correlated, Gaussian errors in the supplementary materials.) We assume that the outcome is an undesirable behavior, so that a decrease in level is desirable.

\footnotetext{
${ }^{2} \mathrm{Bias}$ from using the sample standard deviation is less likely to present problems for the other effect size estimators because $L R R_{1}$, NAP, and Tau do not use $s_{A}$ at all, and $L R R_{2}$ uses it only in the small-sample correction term.
} 

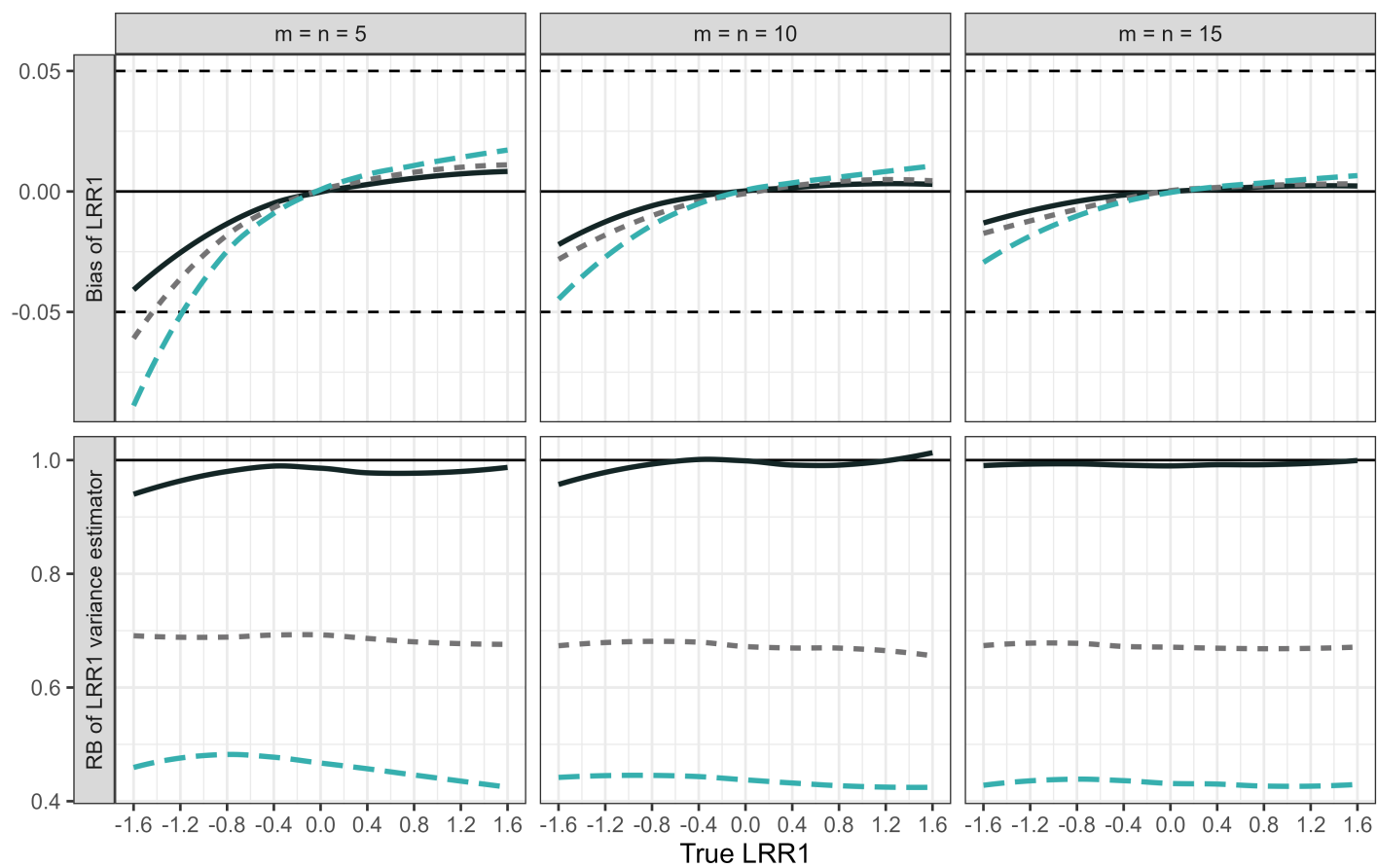

Auto-correlation $-0=-0.2-\cdot 0.4$

Figure 1. Bias of the LRR1 estimator and relative bias (RB) of its variance estimator under the Poisson AR(1) model.

Serially correlated frequency data can be generated using models described by McKenzie (1988). We focus on a first-order auto-regressive model with non-negative autocorrelation parameter $\phi$, where $0 \leq \phi<1$. The $\operatorname{AR}(1)$ Poisson model describes an outcome series $Y_{1}, \ldots, Y_{m+n}$ where $Y_{i}$ is marginally Poisson-distributed with mean $\mu_{A}$ for $i=1, \ldots, m$ and mean $\mu_{B}$ for $i=m+1, \ldots, m+n$. Within each phase, outcomes are correlated according to $\operatorname{corr}\left(Y_{h}, Y_{i}\right)=\phi^{|h-i|}$ for $h, i=1, \ldots, m$ or $h, i=m+$ $1, \ldots, m+n .^{3}$ We simulated single data series based on this model, following the algorithm outlined in Swan \& Pustejovsky (2018).

We simulated data series consisting of one baseline phase and one intervention phase, with a baseline mean of $\mu_{A}=$ 10 and an intervention phase mean determined by the magnitude of the effect size parameter (see the Appendix for further details). We considered data series with equal phase lengths of 5, 10, or 15 observations each and autocorrelation of $\phi=0.0,0.2$, or 0.4 , consistent with past empirical reviews of auto-correlation in SCED data. We varied the effect size parameter across a wide range and simulated $10^{4}$ replications per condition. The simulation code and complete numerical results are available on the Open Science Framework (Chen \& Pustejovsky, 2021b).

LRR. Figures 1 and 2 show the bias of the $L R R_{1}$ (Equation (2)) and $L R R_{2}$ estimator (Equation (3)), re- spectively, and the relative bias of variance estimator (Equation (4)) for LRR parameters ranging from $\lambda=$ -1.6 to 1.6 . Figure 1 illustrates that the $L R R_{1}$ estimator has small negative bias for $\lambda<0$ and slight positive bias for $\lambda>0$. Biases are worse for short phase lengths and large auto-correlation values. Figure 2 shows that, when auto-correlation is absent, the $L R R_{2}$ estimator is nearly unbiased for true effect sizes across the range examined, even for phase lengths as short as 5 observations. For very short phase lengths and for positive auto-correlation, the $L R R_{2}$ estimator has a small bias, which is more pronounced for negative values of $\lambda$ and for stronger levels of auto-correlation. Both $L R R_{1}$ and $L R R_{2}$ estimators are more biased for negative versus positive parameter values because of the difference in the average outcome level for phase $B$ when the frequency counts outcomes are assumed to follow a Poisson AR(1)

\footnotetext{
${ }^{3}$ The correlation between pairs of outcomes in different phases will follow the same structure so long as $\phi \leq \mu_{B} / \mu_{A}$. If there is a large reduction in mean level from baseline to treatment, so that $\phi>\mu_{B} / \mu_{A}$, then the correlation between pairs of observations in different phases can become somewhat distorted. However, this distortion is unimportant because we are not interested in estimating the degree of auto-correlation. Rather, we are interested in how the effect size statistics and associated sampling variances are affected when the datagenerating model includes un-modeled auto-correlation.
} 


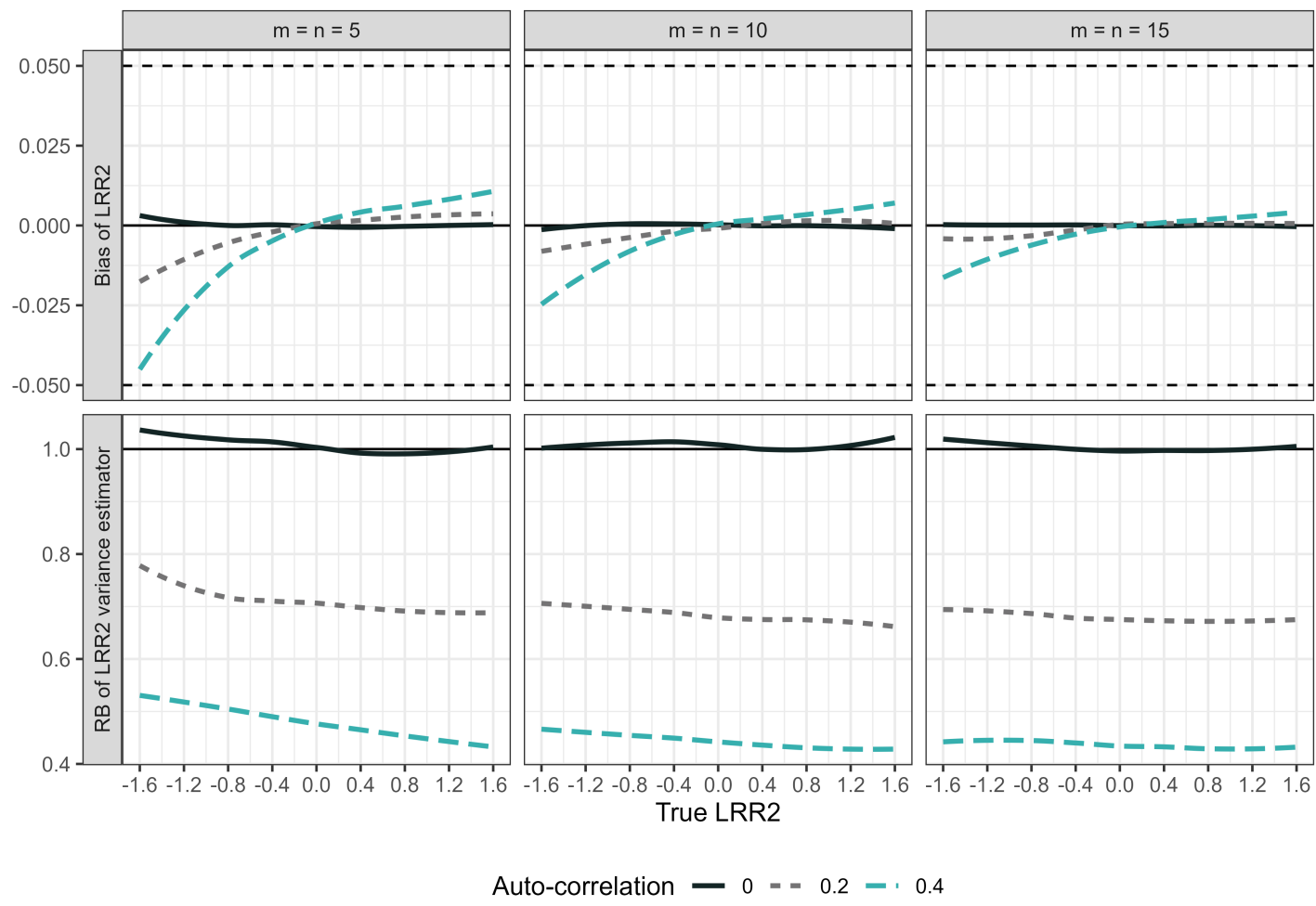

Figure 2. Bias of the LRR2 estimator and relative bias (RB) of its variance estimator under the Poisson AR(1) model.

distribution. For example, when $\lambda=-1.6$, the average frequency count for phase $B$ is very small, and the sampling error for such a low frequency count leads to larger bias estimation of $\log \left(\mu_{B}\right)$ and hence larger bias in the effect size estimators. However, when $\lambda=1.6$, the average frequency count for phase $B$ is very large, and the sampling error for a high frequency count tends to have small or negligible consequences for estimation of $\log \left(\mu_{B}\right)$ and the effect size parameter.

For both estimators, the variance estimator is approximately unbiased when the data are generated independently. However, the variance estimator tends to understate the actual sampling variance in the presence of autocorrelation, and the degree of downward bias increases with strength of auto-correlation. Overall, the $L R R_{2}$ estimator performs better than the $L R R_{1}$ estimator across different degrees of auto-correlation, in terms of bias of the parameter estimator and relative bias of the variance estimator. The same general patterns regarding effect size and variance estimator bias also hold under the Gaussian AR(1) model (see Supplementary Section S2.1).

WC-SMD. Figure 3 displays the bias of the WC-SMD estimator (Hedges' $g$; Equation (6)) and the relative bias of the corresponding variance estimator (Equation (7)) for effect size parameters ranging from -1.6 to 1.6. As shown in the top row, the WC-SMD estimator has a small, positive bias of less than 0.05 when the data are generated without auto-correlation. However, the estimator has a multiplicative bias in the presence of autocorrelation, especially when the number of observations per phase is small ( $m=n=5)$. Longer phase lengths mitigate the bias caused by auto-correlation. The bottom row of Figure 3 demonstrates that the sampling variance of $g$ is under-estimated for most conditions-even in the absence of auto-correlation. As expected, the variance estimator has a strong downward bias for positive values of auto-correlation. Very similar patterns hold under the Gaussian AR(1) model (see Supplementary Section $\mathrm{S} 2.2$ ).

Tau. Figure 4 illustrates the bias of Tau estimator (Equation (11)) and the relative bias of its variance estimator (Equation (12)) for effect size parameters ranging from -0.9 to 0.9 . The top row shows that the Tau estimator is approximately unbiased across the full range of parameter values, even in the presence of auto-correlation. The bottom row shows that the variance estimator is close to unbiased for most scenarios when the data are generated without auto-correlation. However, for short phase lengths $(m=n=5)$ and Tau parameter greater than 0.5 , the variance estimator is increasingly biased and this true for different levels of auto-correlation. This bias arises because we truncated the variance estimator to ensure it is strictly positive, while the true sampling 

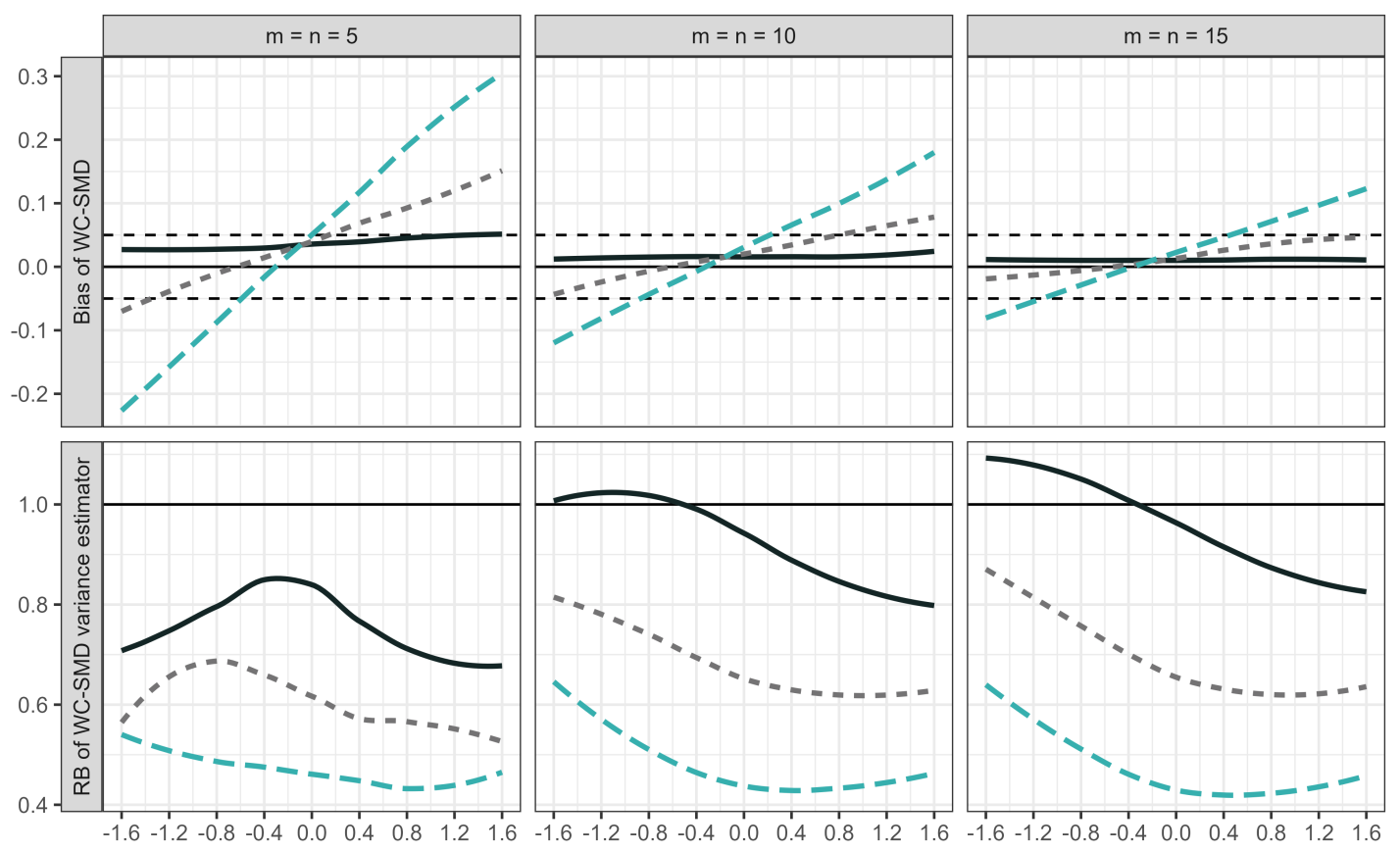

True WC-SMD

Auto-correlation $-0=-0.2=\cdot 0.4$

Figure 3. Bias of the WC-SMD estimator and relative bias (RB) of its variance estimator under the Poisson AR(1) model.

variance decreases to zero as Tau approaches $1 .{ }^{4}$ In the presence of auto-correlation, the variance estimator tends to under-estimate the actual sampling variances for most conditions and higher auto-correlation leads to stronger downward bias. The exception is that the variance estimator has positive bias for large Tau parameter values (i.e., greater than 0.6) when the phase length is short $(m=n=5)$ and this positive bias remains when data are auto-correlated. Generally, the behavior of the Tau effect size estimator and its variance estimator remains very similar when the outcomes follow the Gaussian AR(1) model (see Supplementary Section S2.3).

Remarks. The effect size simulation results demonstrate that the sampling variances of LRR, WC-SMD, and Tau index are generally under-estimated in the presence of auto-correlation. This could lead to bias in the estimation of the variance components and standard error of the overall average effect size in MLMA. Additionally, the $L R R_{1}$ and WC-SMD estimators are biased in the presence of auto-correlation, which could create bias in the overall average effect size estimated via MLMA. ${ }^{5} \mathrm{Be}$ fore examining the consequences of auto-correlation for meta-analysis of SCEDs, we first describe the technical details of the MLMA model and an alternative method of synthesizing effect sizes.

\section{Methods for meta-analysis of SCEDs}

MLMA has been proposed for synthesizing effect size estimates calculated for each case within each study (Pustejovsky \& Ferron, 2017; Van den Noortgate \& Onghena, 2008). The MLMA model can be used in syntheses of SCED studies with different outcome measures, and thus may be more broadly applicable than one-stage, raw-data synthesis approaches. Moreover, the MLMA model accounts for the hierarchical structure where caselevel effect sizes are nested within studies. However, the conventional standard errors and hypothesis tests based

\footnotetext{
${ }^{4}$ As shown in Supplementary Section S1.1, the true sampling variance of Tau decreases to zero as the Tau parameter approaches 1. As shown in the Supplementary Section S1.2, the non-truncated variance estimator has only slight bias for short phase lengths and extreme Tau parameters.

${ }^{5}$ In addition to the biases of the effect size estimators and variance estimators, we also examined their sampling variances and correlation. In Supplementary Section S1.1, we show that the sampling variance of each of the effect size estimators is generally related to the magnitude of the effect size parameter and that, for non-null effects, the variance estimators tend to be correlated with their corresponding effect size estimators. Both of these properties have implications for use of the multi-level meta-analytic model, as described in the following section.
} 

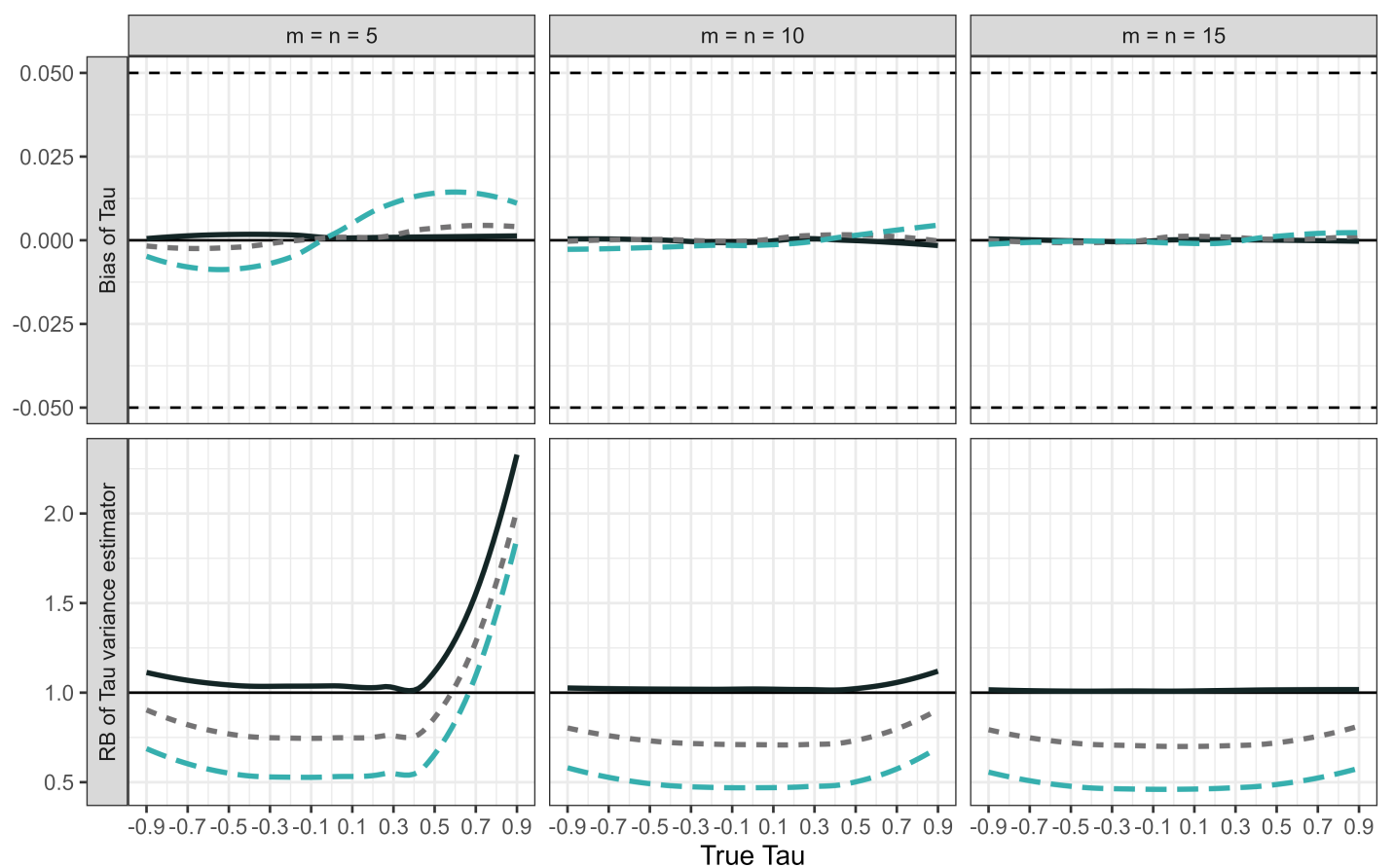

Auto-correlation $-0=-0.2=\cdot 0.4$

Figure 4. Bias of the Tau estimator and relative bias (RB) of its variance estimator under the Poisson AR(1) model.

on MLMA may be sensitive to mis-estimation of the effect size sampling variances, as would occur if the outcome data series is auto-correlated. Although MLMA has been widely applied in practice, its performance in the presence of auto-correlation has yet to be evaluated.

Robust variance estimation methods present a potential solution to the auto-correlation issue. RVE has been widely adopted for meta-analysis of between-groups designs (Hedges et al., 2010). Pustejovsky (2018) proposed the use of RVE in combination with MLMA of SCEDs to address the possibility that the sampling variances of effect sizes might be estimated inaccurately. Theoretically, using RVE in this way should be expected to produce standard errors that take into account the possibility of auto-correlation of within-case errors and the dependence structure arising from having multiple effect size estimates nested within studies (Pustejovsky, 2018).

Although RVE may be useful for correcting standard errors of the overall average effect size estimates in MLMA, it is not a panacea for all violations of model assumptions. In particular, violation of certain assumptions of the MLMA model may lead to bias in estimation of overall average effect sizes. A simpler alternative to the MLMA is ordinary least squares, or un-weighted averaging of effect sizes, which relies on weaker assumptions than MLMA. Thus, it is important to assess whether OLS might perform acceptably well in terms of estimating the overall average effect sizes under conditions where MLMA might not work properly.

In this section, we first review the MLMA model, its assumptions, and its use with RVE (MLMA-RVE). We then describe the OLS approach and its use in conjunction with RVE (OLS-RVE). We consider a meta-analytic database of $K$ single-case studies, where study $k$ includes $J_{k}$ distinct cases. Let $T_{j k}$ denote the within-case effect size estimator (LRR, WC-SMD, or Tau) and let $\gamma_{j k}$ denote the true effect size parameter for case $j$ in study $k$, where $j=1,2, \ldots, J_{k}$ and $k=1,2, \ldots, K$. Let $e_{j k}$ be the sampling error of the effect size estimator. Following convention, we assume that the effect size estimator is unbiased and that its sampling error has known variance $V_{j k}$.

\section{MLMA}

The MLMA can be understood as a hierarchical model with three levels, corresponding respectively to the sampling level, the case level, and the study level. At the sampling level, we model the effect size estimator as,

$$
T_{j k}=\gamma_{j k}+e_{j k} \quad \text { where } e_{j k} \sim N\left(0, V_{j k}\right) .
$$

Let $\gamma_{k}$ be the average effect size parameter for study $k$, $u_{j k}$ be the case-specific deviation from the average, and $\omega$ 
be the standard deviation of $u_{j k}$, capturing within-study heterogeneity. At the case level, we model the true effect size parameter for case $j$ from study $k$ as

$$
\gamma_{j k}=\gamma_{k}+u_{j k} \quad \text { where } \quad u_{j k} \sim N\left(0, \omega^{2}\right) .
$$

To complete the model, let $\gamma$ be the overall average effect size parameter, $v_{k}$ be a study-level error term, and $\tau$ be the standard deviation of $v_{k}$, capturing between-study heterogeneity. At the study level, we assume

$$
\gamma_{k}=\gamma+v_{k} \quad \text { where } \quad v_{k} \sim N\left(0, \tau^{2}\right) .
$$

Combining all three levels together, the MLMA model has the form

$$
T_{j k}=\gamma+v_{k}+u_{j k}+e_{j k}
$$

(Konstantopoulos, 2011; Van den Noortgate \& Onghena, 2008). Note that $V_{j k}, e_{j k}, u_{j k}$, and $v_{k}$ are assumed to be mutually independent. This basic MLMA model can be extended to include predictors in a meta-regression, although we do not consider this possibility here. ${ }^{6}$

There are several ways in which the assumptions of the MLMA could be violated when applied to withincase effect sizes for SCEDs. First, the sampling variances $V_{j k}$ are derived based on the assumption that the outcome measurements are mutually independent within each phase. If this assumption is violated, $V_{j k}$ will be an inaccurate estimator of the true sampling variance, as demonstrated in the previous section, which may lead to inaccurate statistical inference about the overall average effect size. Second, treating the sampling variances as known constants might be inappropriate if the estimators are calculated based on a small number of observations. Third, if the effect size estimators are themselves biased, then the sampling errors $e_{i j}$ will not have mean zero, and the bias may be propagated through to the meta-analytic average effect size estimator. Finally, the assumption that the model errors are independent of the sampling variance $V_{j k}$ may be violated for some effect size measures, particularly if the sampling variance estimator $V_{j k}$ is correlated with either the effect size estimator $T_{j k}$ or with the true effect size parameter $\gamma_{j k}$.

Estimation. Estimation of the MLMA model parameters involves first obtaining estimates of the case-level and study-level heterogeneity parameters, $\omega$ and $\tau$, and then estimating the overall average effect size $\gamma$ (Pustejovsky, 2018). The heterogeneity parameters are typically estimated using maximum likelihood estimation or restricted maximum likelihood estimation methods, which are available in statistical analysis software packages such as SAS or R. Let $\hat{\omega}$ and $\hat{\tau}$ represent the estimators of $\omega$ and $\tau$, respectively. The estimators $\hat{\omega}$ and $\hat{\tau}$ are used for estimating the overall average effect size and its standard error.

Given heterogeneity parameter estimators, the overall average effect size is estimated by taking a weighted average of the case-specific effect size estimators. The effect size for study $k, \gamma_{k}$, is estimated using a weighted average of the effect size estimators for all cases in study $k$ :

$$
\hat{\gamma}_{k}=\frac{1}{W_{k}} \sum_{j=1}^{n_{k}} w_{j k} T_{j k}, \quad \text { where } \quad w_{j k}=\frac{1}{\hat{\omega}^{2}+V_{j k}}
$$

and $W_{k}=\sum_{j=1}^{n_{k}} w_{j k}$. The overall average effect size is then calculated as

$$
\hat{\gamma}=\frac{1}{H} \sum_{k=1}^{K} h_{k} \hat{\gamma}_{k}, \quad \text { where } \quad h_{k}=1 /\left(\hat{\tau}^{2}+\frac{1}{W_{k}}\right)
$$

and $H=\sum_{k=1}^{K} h_{k}$ (Pustejovsky, 2018). Under the assumptions of the MLMA model, the variance of the overall average effect size can be approximated by $1 / H$ (Pustejovsky, 2018)—we call this the conventional variance estimator. The hypothesis test of the overall average effect size and the corresponding confidence interval are based on a $t$-distribution with $N-1$ degrees of freedom, where $N$ indicates the total number of effect size estimates across all studies.

Equation (17) reveals how violation of the MLMA model assumptions can lead to bias. Specifically, if $T_{j k}$ is biased or if $T_{j k}$ is correlated with $w_{j k}$, then $\hat{\gamma}_{k}$ will be a biased estimator of the true study-level average effect size $\gamma_{k}$. If this bias or correlation exists across studies, then this bias will be propagated through to the the overall average effect size estimator.

\section{RVE}

The conventional, model-based variance under the MLMA might be biased when the sampling variances of effect sizes $\left(V_{j k}\right)$ or the heterogeneity estimates $(\hat{\tau}$ and $\hat{\omega})$ are not accurate, as could be the case if the outcome data are auto-correlated. An alternative approach uses RVE methods, which do not rely on the accuracy of effect size sampling variances and variance component estimates in estimating the variance of overall average effect size estimate. The robust variance estimator for $\hat{\gamma}$ is calculated as

$$
V^{C R}=\frac{1}{H^{2}} \sum_{k=1}^{K} \frac{h_{k}^{2}\left(\hat{\gamma}_{k}-\hat{\gamma}\right)^{2}}{1-h_{k} / H}
$$

\footnotetext{
${ }^{6}$ The meta-regression model incorporates one or more participant or study characteristics as predictors of average effect sizes. For example, Pustejovsky (2018) examined the extent to which study setting (general or special education class) and unit of analysis (group-level or individual) moderate intervention effects in SCED studies on group-contingency interventions.
} 
(Pustejovsky, 2018). This variance estimator incorporates a small-sample correction to improve its accuracy when the total number of studies $K$ is limited (Tipton, 2015). The hypothesis test of the overall average effect size and the corresponding confidence interval are based on the small-sample corrected robust standard errors and a $t$-distribution with Satterthwaite degrees of freedom.

\section{OLS with RVE}

For estimating the overall average effect size, a simple alternative to the MLMA model is to use ordinary least squares (OLS) estimation (i.e., take the simple average). Following the notation above, the OLS approach involves estimating an intercept-only regression model

$$
T_{j k}=\gamma+\varepsilon_{j k},
$$

where $\varepsilon_{j k} \sim N\left(0, \sigma^{2}\right)$. The overall average effect size is estimated with the simple average of effect sizes across cases and studies

$$
\bar{\gamma}=\frac{1}{N} \sum_{k=1}^{K} \sum_{j=1}^{J_{k}} T_{j k}
$$

where $N=\sum_{k=1}^{K} J_{k}$ is the total number of effect sizes. Because OLS uses the simple (un-weighted) average of the effect size estimators, it avoids the potential for bias arising from correlation between the effect size estimators and the MLMA weights. This does comes at a cost, though. If the MLMA modeling assumptions are reasonable, then we would expect the un-weighted average to be less precise than the weighted average based on the MLMA.

The usual variance estimator for $\bar{\gamma}$ is based on the assumption that the errors $\varepsilon_{j k}$ are mutually independent and have equal variance. This assumption will typically be violated because effect size estimates from the same study will tend to be correlated, rather than independent, and may also vary in their precision. To handle violation of these assumptions, a robust variance estimator for $\bar{\gamma}$ can be calculated as

$$
V_{o l s}^{C R}=\frac{1}{N^{2}} \sum_{k=1}^{K} \frac{J_{k}^{2}\left(\bar{\gamma}_{k}-\bar{\gamma}\right)^{2}}{1-J_{k} / N},
$$

where $\bar{\gamma}_{k}=\sum_{j=1}^{J_{k}} T_{j k} / J_{k}$. The hypothesis test and the confidence interval for the overall average effect size are based on a $t$-distribution with Satterthwaite degrees of freedom. $^{7}$

The meta-analytic approaches that we have reviewed here rely on different assumptions and yield average effect size estimates with differing precision when their assumptions hold. RVE may be useful in addressing some violations of the MLMA modeling assumptions. Other violations may lead to bias in the average effect size estimator from MLMA, which could be avoided by using OLS. Furthermore, different effect size metrics have different properties (with respect to bias, sampling variance estimation, and correlation between effect size and sampling variance), making it difficult to gauge a priori which meta-analytic approach should be preferred. Thus, we conducted a simulation study to evaluate the performance of the MLMA and OLS approaches for metaanalysis of SCEDs and to examine the performance of RVE in handling the violation of model assumptions, for each of three different effect size metrics.

\section{Simulation methods}

We conducted a Monte Carlo simulation study to evaluate the performance of MLMA, with and without RVE, and OLS with RVE in estimating the average treatment effects when the outcome data are auto-correlated. We ran the simulation in $\mathrm{R}$ Version 4.0.2 ( $\mathrm{R}$ Core Team, 2020). Code for replicating the simulations and complete numerical simulation results are provided on the Open Science Framework (Chen \& Pustejovsky, 2021b).

\section{Data-generating model}

We simulated meta-analytic data for SCEDs with LRR, WC-SMD, or Tau as the effect size measure. We followed the strategy of generating raw data from multiple SCEDs and calculating effect size estimates based on the generated raw data, a process involving several steps. First, we simulated effect size parameters for each case. Second, we simulated several auxiliary parameters that describe features of the study designs. Third, based on the auxiliary parameters and effect size parameters, we generated raw data for each case and calculated an effect size estimate and its sampling variance. Finally, we estimated meta-analytic models based on the effect size estimates and their sampling variances. We now explain these steps in detail.

Simulating effect size parameters for each case. We generated meta-analytic data for $K$ primary studies, where study $k$ included $J_{k}$ cases. We used a metaanalytic model to generate effect size parameters for case $j\left(j=1, \ldots, J_{k}\right)$ in study $k(k=1, \ldots, K)$. We simulated the number of cases per study by sampling $J_{k}$ at random from the integers 1 to 5 . This resulted in an average of 3 cases per study, consistent with findings from

\footnotetext{
${ }^{7}$ The Satterthwaite degrees of freedom in OLS-RVE are different than those from MLMA-RVE because the variance estimators are different. Tipton (2015) provides further details about the Satterthwaite approximation.
} 
empirical reviews that the number of individual participants in single-case studies typically ranges from 2 to 4 with mean and median near 3 (Pustejovsky et al., 2019; Shadish \& Sullivan, 2011). We simulated effect size parameters separately for each effect size metric.

Simulating the LRR and WC-SMD effect size parameters involved similar steps. Given parameters of the MLMA model, we simulated the average LRR effect size parameter $\gamma_{k}$ for study $k$ using Equation (15), then simulated the case-specific LRR effect size parameter $\gamma_{j k}$ for case $j$ in study $k$ using Equation (14). The generation of case-specific WC-SMD effect size parameters followed the same process.

The generation of meta-analytic data for Tau required a different process because the range of the effect size parameter is restricted to -1 and $1 .{ }^{8}$ We generated data based on a convolution of shifted-and-scaled beta distributions (over the range -1 to 1 ), with mean and variance structure that approximated the MLMA model (Equations (14) and (15)) as closely as possible. First, we generated study-specific average effect size parameters $\lambda_{k}$ based on a shifted-and-scaled beta distribution with mean $\gamma$ and variance $\tau^{2} .{ }^{9}$ Next, we generated the casespecific effect size parameters $\gamma_{j k}$ for study $k$ based on a shifted-and-scaled beta distribution with mean $\theta_{k}$ and variance of approximately $\omega^{2}$. The beta distribution has the property that $\theta_{k}^{2}<1-\omega^{2}$, because of which it was not always possible to simulate case-specific effect size parameters. To address this constraint, we set $\gamma_{j k}$ to be equal to $\gamma_{k}$ if $\theta_{k}^{2} \geq 1-\omega^{2}$.

Simulating auxiliary parameters. Simulating raw data for a single series requires specifying the mean levels of phases $\mathrm{A}$ and $\mathrm{B}, \mu_{A}$ and $\mu_{B}$, the number of observations in each phase, $m$ and $n$, and the auto-correlation $\phi$. We sought to simulate values for $\mu_{A}, m$, and $n$ in a realistic way, informed by the empirical features of real SCED data. For instance, we allowed the number of observations in the baseline phase to differ from the number in the intervention phase because real SCDs often have phases of unequal length.

Pustejovsky et al. (2019) examined the measurement procedures and characteristics of baseline data in a corpus of over 300 single-case studies with behavioral outcomes. For frequency count outcomes of undesirable behaviors, they found that the average baseline level of 11 events, with an interquartile range of 4.9 to 24 . Based on these findings, we simulated the baseline mean level $\mu_{A}$ for study $k$ using a truncated gamma distribution with

$$
\mu_{A} \sim \max \{5, \Gamma(\alpha=2, \beta=7)\},
$$

where $\alpha$ is the shape parameter and $\beta$ is the scale parameter. We truncated the gamma distribution at 5 in order to avoid restricting the range of possible Tau parameters; see the Appendix for further details regarding the range restriction issue.

We generated the number of data points in each phase, $m$ and $n$, with a shifted Poisson distribution

$$
m \sim 3+\text { Poisson(4), } \quad n \sim 3+\text { Poisson(4) }
$$

This resulted in a median of 7 data points per phase, with a mean of approximately 7. This value was selected on the basis of the findings in Pustejovsky et al. (2019), which showed the initial baseline phases having from 5 to 12 data points with a median of 7 . The minimum number of data series in baseline was set to 3 , consistent with the design standards of the What Workings Clearinghouse (What Works Clearinghouse, 2020) and Council for Exceptional Children (Cook et al., 2014).

Simulating raw data. Given values of the casespecific effect size parameters $\gamma_{j k}$ and the case-specific baseline mean $\mu_{A}$, we determined the intervention phase mean $\mu_{B}$ as described in the Appendix. Then, given the case-specific values of $m$ and $n$ and the auto-correlation $\phi$, we simulated raw data from the auto-correlated Poisson model, following the same process as in the effect size simulations described in a previous section. We then calculated effect size estimates and associated sampling variances from the raw data, yielding a meta-analytic dataset.

In all, our data-generating process emulates many features of real SCED data, although it does involve some degree of abstraction. In particular, we did not directly simulate the data structures of single-case designs such as multiple baseline designs or treatment reversal (ABAB) designs. Still, our process yields data that resemble the structural features of real designs. The number of cases in a given study was randomly selected from the integers between 1 and 5. For studies with three or more cases, phase lengths were randomly generated for each case, and so the simulated data can be interpreted as coming from a multiple baseline design with randomly selected intervention times. For studies with just one or two cases, the simulated data can be interpreted as

\footnotetext{
${ }^{8}$ For Poisson-distributed random variables $Y^{A}$ and $Y^{B}$, where a decrease in the outcome is desirable, the maximum possible value of the Tau parameter is restricted to values less than 1 and is a function of $\mu_{A}=\mathrm{E}\left(Y^{A}\right)$. Smaller values of $\mu_{A}$ lead to smaller upper limits on the range of Tau. See the Appendix for details. For purposes of simulation, we avoided this range restriction by setting $\mu_{A} \geq 5$.

${ }^{9}$ For a shifted-and-scaled beta distribution with support (-1, 1 ), mean $\gamma$, and variance $\tau^{2}$, the parameter space is restricted to $\tau^{2}<1-\gamma^{2}$. For purposes of simulation, we chose parameter values that satisfied this constraint.
} 
Table 1

Data generating conditions for meta-analytic data simulation.

\begin{tabular}{ll}
\hline Conditions & \multicolumn{1}{c}{ Values } \\
\hline Effect size parameters $(\gamma)$ & LRR: $-1.6,-1.2,-0.8,-0.4,0.0,0.4,0.8,1.2,1.6$ \\
& WC-SMD: $-1.6,-1.2,-0.8,-0.4,0.0,0.4,0.8,1.2,1.6$ \\
& Tau: $-0.8,-0.6,-0.4,-0.2,0.0,0.2,0.4,0.6,0.8$ \\
Number of studies $(K)$ & 10,30 \\
Between-study heterogeneity $(\tau)$ & $0.1,0.3$ \\
Within-study heterogeneity $(\omega)$ & $0.1,0.2$ \\
Auto-correlation $(\phi)$ & $0.0,0.2,0.4$ \\
\hline
\end{tabular}

the initial A and B phases from a treatment reversal design with randomly selected phase changes. Although the generated raw data might not exactly reflect the complex data structure in meta-analysis of SCEDs, we believe that this data generating process is adequate for an initial investigation of the performance of MLMA and RVE under different conditions.

\section{Estimation methods}

For each simulated meta-analytic dataset, we used OLS and MLMA for estimating the overall average effect size. The OLS estimator was applied using the $\operatorname{lm}($ ) function from the stats package. The MLMA model was applied using rma. mv ( ) from the meta for package (Viechtbauer, 2010), with heterogeneity parameters estimated by restricted maximum likelihood. We applied RVE to the model results using the coef_test ( ) and coef_inf () functions from the clubsandwich package (Pustejovsky, 2020).

\section{Experimental design}

We studied the performance of the meta-analytic estimation methods across a range of parameter values, using a $9 \times 3 \times 2 \times 2 \times 2 \times 3$ design, for a total of 648 conditions. Table 1 summarizes the design of the simulation.

The average effect size parameters varied from -1.6 to 1.6 in steps of 0.4 for LRR and WC-SMD and from 0.8 to 0.8 in steps of 0.2 for Tau. These ranges allowed us to examine performance across a broad range of true effect sizes for each effect size measure. Note that Tau has different range than LRR and WC-SMD because it is bounded in the interval -1 to 1 . The number of studies $K$ was set to 10 or 30 , consistent with the values used in the simulation study of Moeyaert et al. (2013). The auto-correlation parameter, $\phi$, was set to $0.0,0.2$, or 0.4 , consistent with existing empirical evidence about levels of auto-correlation in SCED data (Shadish \& Sullivan, 2011; Solomon, 2014).
The values for between-study heterogeneity and withinstudy heterogeneity were selected based on empirical evidence from two meta-analyses of SCEDs with behavioral outcomes. First, Pustejovsky (2018) reanalyzed data from a meta-analysis of SCED studies on group-contingency interventions (Maggin, Pustejovsky, \& Johnson, 2017), using LRR as the effect size metric. The estimated MLMA model indicated betweenstudy heterogeneity of 0.4 and within-study heterogeneity of 0.2 . In a meta-analysis of the effects of peer reporting interventions on disruptive behaviors (Collins et al., 2020), between-study heterogeneity was estimated as 0.4 for LRR and 0.3 for Tau for the disruptive behaviors; within-study heterogeneity was estimated at 0.17 for LRR and 0.1 for Tau for the disruptive behaviors. For simplicity, we chose the same sets of between-study heterogeneity $\tau=(0.1,0.3)$ and within-study heterogeneity $\omega=(0.1,0.2)$ for all three effect size metrics.

\section{Performance criteria}

We generated 4,000 meta-analytic datasets per condition. For the given effect size metric (LRR, WC-SMD, or Tau), we calculated several performance criteria for the average intervention effect estimates obtained using MLMA model and OLS approach, with and without RVE. First, we calculated bias of the average effect size estimates. Second, we measured the accuracy of the average effect size estimates using root mean-squared error (RMSE). Third, we computed coverage rates of $95 \%$ confidence intervals (i.e., the proportion of replications in which the CI contains the true average effect size parameter). Finally, we assessed Type I error rates of robust hypothesis tests for the subset of conditions where the average effect size parameter was equal to zero. The restricted maximum likelihood estimation for the MLMA model converged very well under most conditions (see Supplementary Section S3.1). The non-convergent cases were not used for calculating these performance criteria for either MLMA or OLS method. 

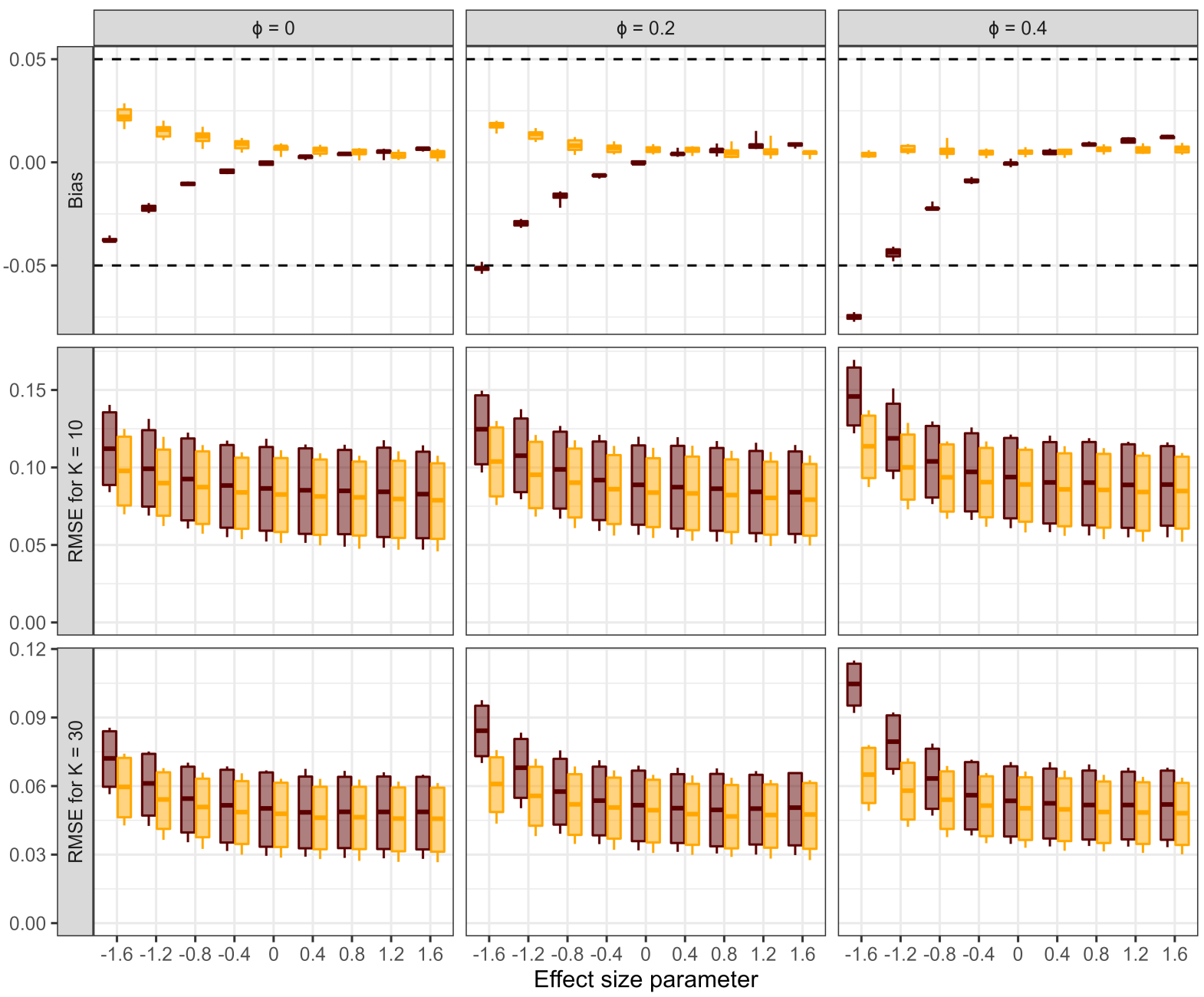

OLS MLMA

Figure 5. Bias and RMSE of the average LRR1 estimates.

\section{Simulation results}

We present the results for each effect size metric in terms of bias for the overall average effect size estimates, RMSE, and CI coverage. We discuss the results for both $L R R_{1}$ and $L R R_{2}$ in order to inform the choice of estimators for use in meta-analysis based on the LRR metric. Supplementary Section S3.2 includes results regarding Type I error rates of each estimation method for each effect size metric.

\section{LRR1}

Figure 5 displays bias (the first row) and RMSE (the second and third row) of the OLS and MLMA estimators using the $L R R_{1}$ effect size estimator for true LRR parameters ranging from -1.6 to 1.6 (the horizontal axis).
Note that we present RMSE for $K=10$ in the second row and for $K=30$ in the third row because the accuracy of $L R R_{1}$ estimator varies with the number of studies in the meta-analysis. In the figure, columns correspond to varying levels of auto-correlation under the AR(1) Poisson model; the left column corresponds to no auto-correlation-theoretically, the most favorable condition for the MLMA model without RVE; the middle and right columns correspond to conditions with moderate or stronger auto-correlation, where the sampling variances of the effect sizes will be under-estimated. Within each graph, individual boxplots show the range of bias or RMSE across the remaining simulation conditions (e.g., RMSE across levels of between-study and within-study heterogeneity).

Bias. The top row of Figure 5 shows that OLS estimator for the average LRR is nearly unbiased for zero or 


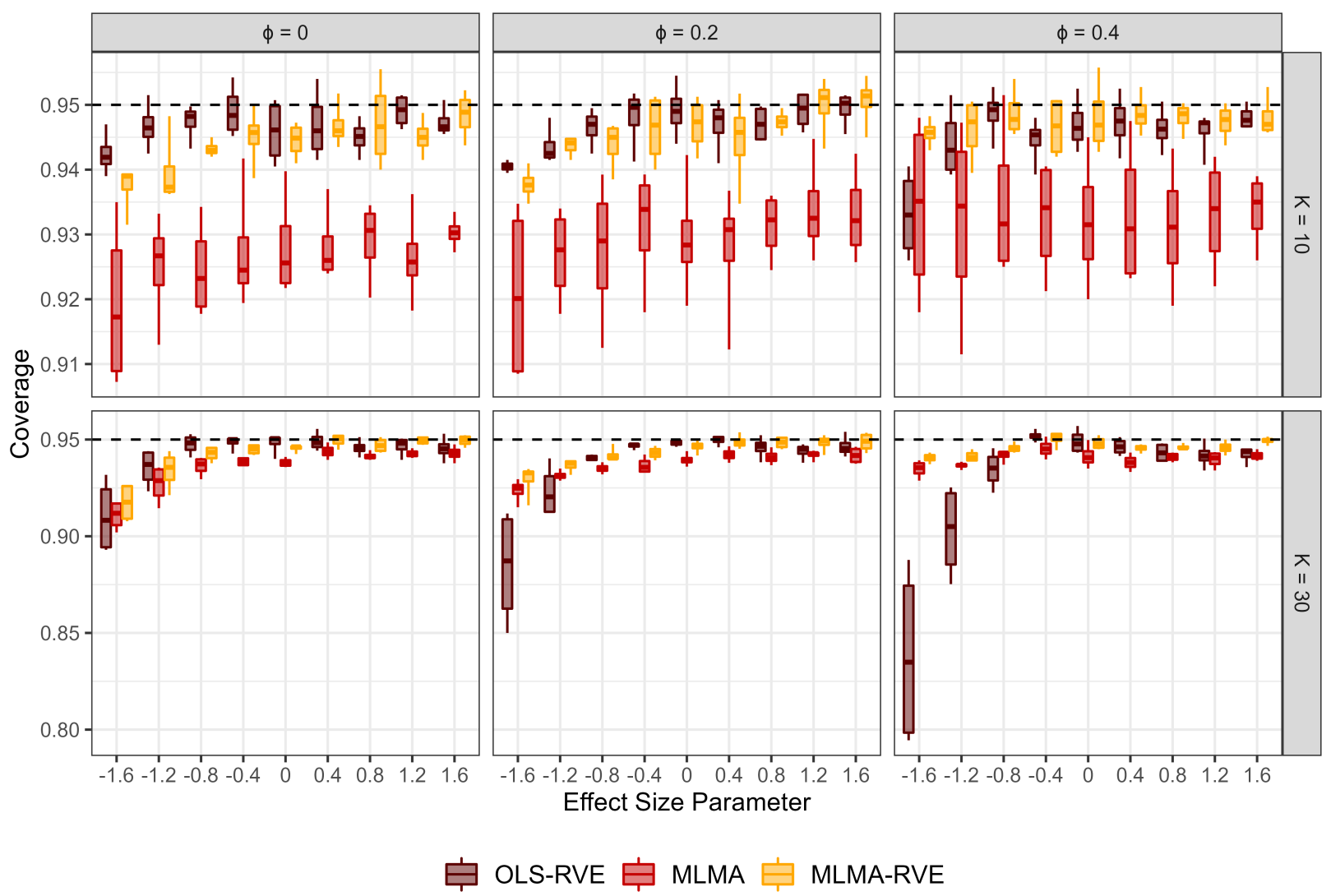

Figure 6. Coverage rate of the confidence intervals for LRR1.

positive parameter values. However, it tends to have negative bias for negative parameter values, which is due to the bias in the case-level effect size estimator. The bias increases for stronger degree of auto-correlation, following the same pattern as the bias of the case-level effect size estimator. The MLMA estimator has small positive bias for negative parameter values, which is reduced for larger values of auto-correlation. Surprisingly, using MLMA seems to mitigate the bias of the case-level $L R R_{1}$ estimator.

Accuracy. As illustrated in the middle and bottom rows of Figure 5, the MLMA estimator is generally more accurate than the OLS estimator, no matter whether the raw data are generated with or without auto-correlation. Across conditions, RMSE of MLMA ranges from 0.53 to 1.00 times the RMSE of OLS, with a median of 0.934 (see Supplementary Section S3.3.1). Both estimators are more accurate for smaller values of auto-correlation and for the larger number of studies.

Confidence interval coverage. We estimated the coverage rates of the $95 \%$ confidence intervals based on OLS-RVE, MLMA (with the conventional variance estimator), and MLMA-RVE. Figure 6 illustrates the CI coverage for $K=10$ (the top row) and $K=30$ (the bot- tom row) across different levels of auto-correlation (the columns). The CI coverage rates based on OLS-RVE are close to the nominal level of 0.95 for small autocorrelation and the smaller number of studies. However, coverage rates of OLS-RVE tend to decrease for large auto-correlation and large number of studies due to the bias of the OLS estimator under these conditions. MLMA with the conventional variance estimator has below-nominal coverage levels when the number of studies is small, although coverage does not seem to be negatively impacted by auto-correlation. The coverage rates of CIs based on MLMA-RVE remain close to nominal levels under most conditions, and generally have closer-to-nominal coverage than conventional MLMA and OLS-RVE-particularly in the presence of autocorrelation. Overall, applying RVE to the MLMA estimator improves performance for meta-analysis of $L R R_{1}$ effect size estimates.

\section{LRR2}

Figure 7 illustrates the bias and RMSE of the OLS and MLMA estimators based on the $L R R_{2}$ effect size estimator, across a wide range of true LRR parameter values. 

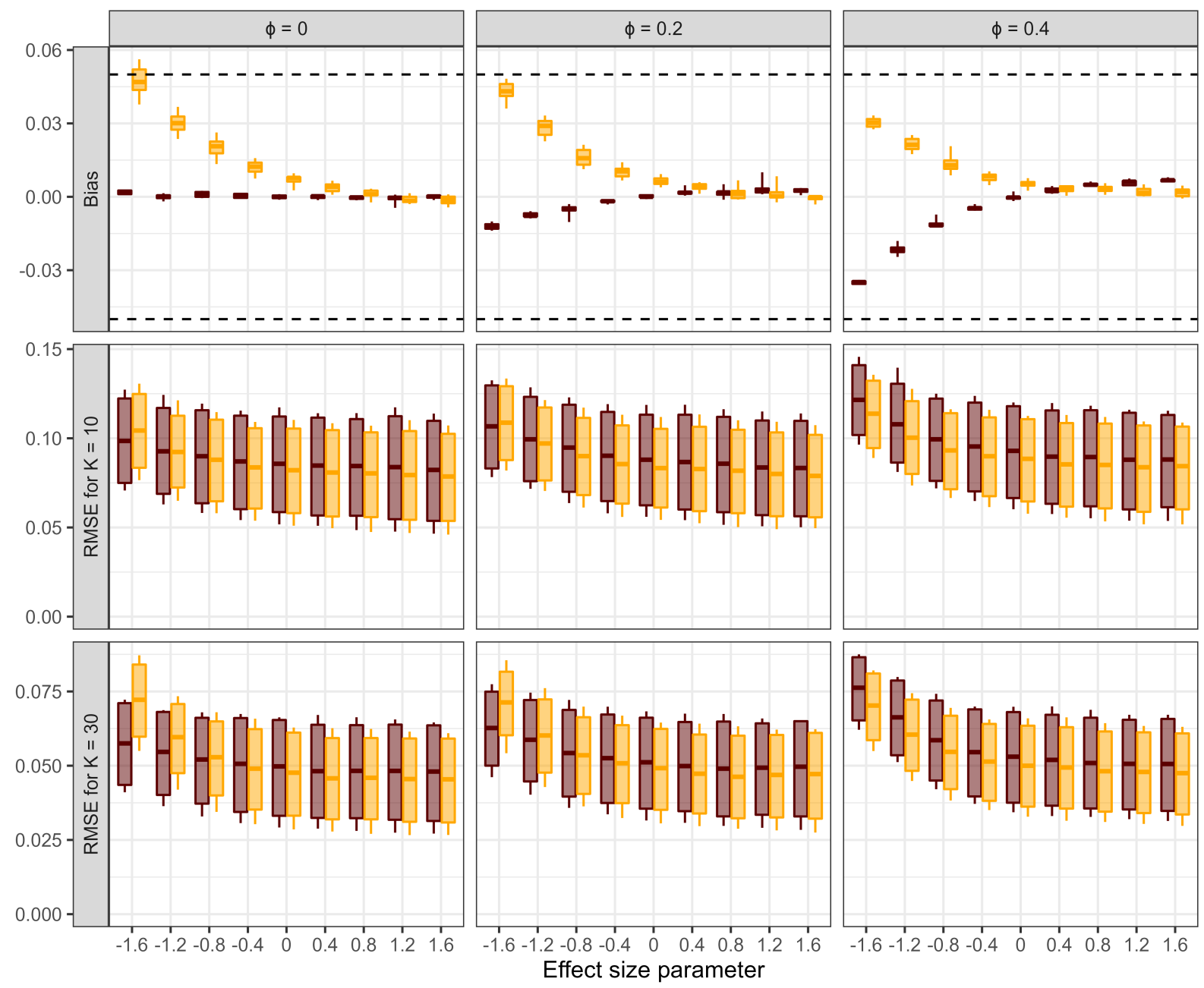

追 OLS 追 MLMA

Figure 7. Bias and RMSE of the average LRR2 estimates.

Bias. Both the OLS and MLMA estimators are close to unbiased for positive true parameters. The OLS estimator is approximately unbiased in the absence of autocorrelation but tends to have small bias in the presence of auto-correlation. The bias is larger for negative parameter values and stronger levels of auto-correlation. The MLMA estimator has a small positive bias in the absence of auto-correlation, which reduces for larger autocorrelation values. Because $L R R_{2}$ is nearly unbiased in the absence of auto-correlation, the bias of the MLMA estimator likely arises because of correlation between the effect size estimator and the weights used in the MLMA average effect estimator.

Accuracy. The MLMA estimator produces slightly more accurate average $L R R_{2}$ estimates than OLS estimator for most scenarios, except when the true LRR parameter are close to -1.6 and auto-correlation is small. The relative RMSE of MLMA over OLS ranges from 0.876 to 1.384 with a median of 0.958 (see Supplementary Section S3.3.1). Both OLS and MLMA estimators are more accurate for larger numbers of studies.

Confidence interval coverage. As shown in Figure 8, the CI coverage rates of OLS-RVE with $L R R_{2}$ effect sizes remain close to the nominal level of 0.95 under most conditions. The CI coverage decreases slightly for large number of studies when the parameter values are extremely negative and when auto-correlation is strong. The CI coverage rates of MLMA-RVE are close to the nominal level when parameter values are greater than 0.4 , but decrease for extreme negative values and larger number of studies, likely due to the bias of the MLMA average effect estimator under these conditions. Compared to conventional MLMA, MLMA with RVE maintains closer-to-nominal coverage across all conditions. 


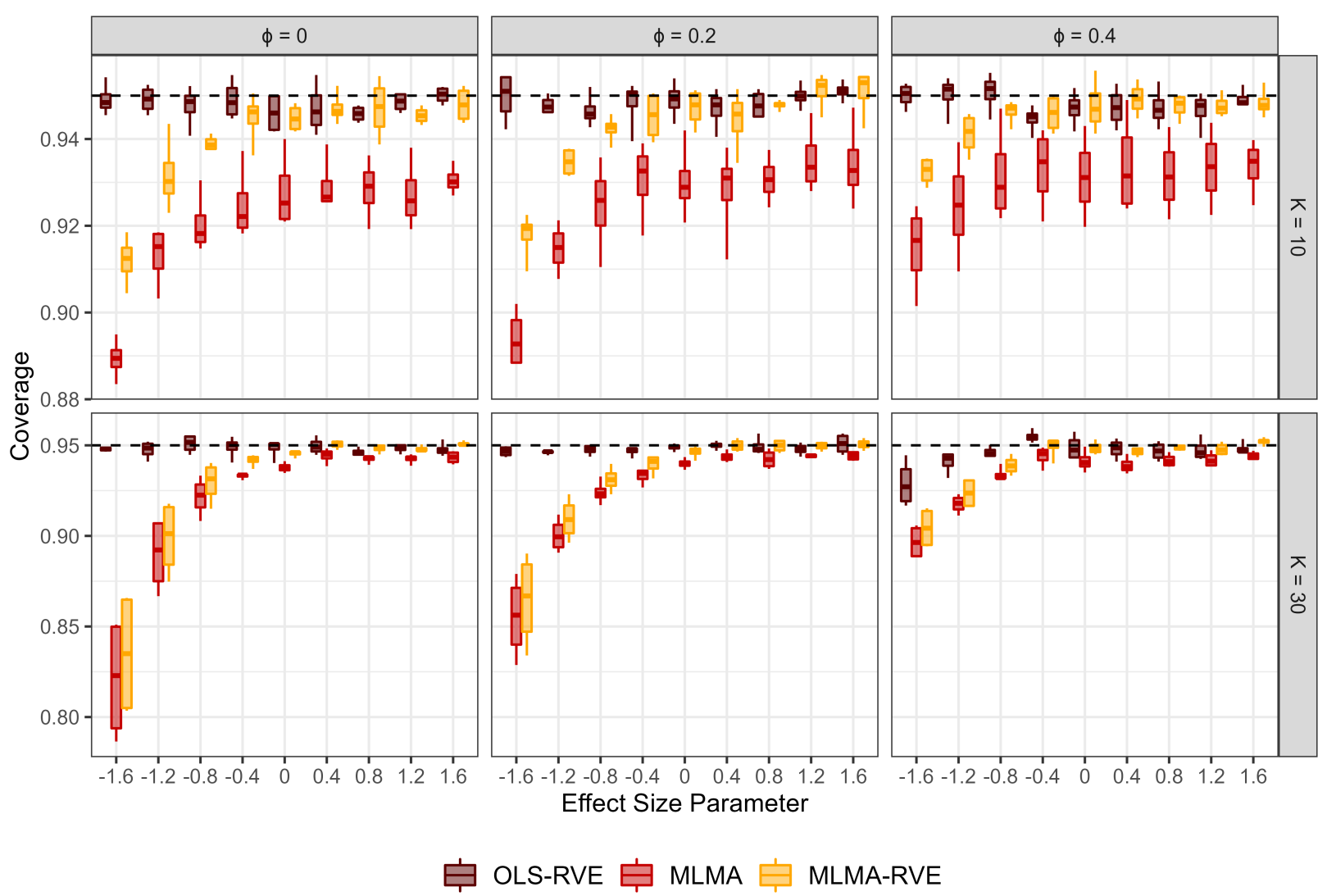

Figure 8. Coverage rate of the confidence intervals for LRR2.

\section{WC-SMD}

Figure 9 depicts the bias and RMSE of the OLS and MLMA estimators for the average WC-SMD across different true effect size values (the horizontal axis) and different auto-correlation values (columns). RMSE is presented for $K=10$ and $K=30$.

Bias. In the top row of Figure 9, the MLMA estimator for the average WC-SMD has a systematic, multiplicative bias, which occurs under conditions with and without auto-correlation. The bias is towards zero (i.e., larger positive effect sizes tend to be more strongly underestimated, larger negative effect sizes tend to be more strongly over-estimated). In contrast, the OLS estimator for the average WC-SMD effect size is close to unbiased when the raw data are generated without autocorrelation. However, the OLS estimator tends to be biased under conditions with positive auto-correlation. Stronger auto-correlation creates a stronger multiplicative bias, so that the absolute magnitude of the average effect size is over-stated.

Accuracy. It can be seen in Figure 9 that the OLS estimator is less accurate (i.e., larger RMSE) for large autocorrelation values and for the smaller number of stud- ies. When $\phi=0$, the OLS estimator produces more accurate average WC-SMD estimates than the MLMA estimator for moderate or large true effects (i.e., smaller than -0.4 or larger than 0.4 ), but less accurate estimates for small effects. However, the accuracy of the OLS estimator tends to decrease with auto-correlation while the accuracy in MLMA tends to increase for large autocorrelation. When $\phi=0.4$, the MLMA estimator yields more accurate average WC-SMD estimates than OLS. Both estimators produce more accurate estimates for the larger number of studies.

Confidence interval coverage. As shown in the top row of Figure 10, OLS-RVE has near-nominal CI coverage rates when the number of studies is small. Its coverage degrades in the presence of auto-correlation, especially when the number of studies is large, due to the bias of the OLS average effect estimator. Still, CIs based on OLS-RVE perform better than those based on MLMA or MLMA-RVE across nearly all conditions. The MLMA estimators, with or without RVE, have coverage rates far below nominal level under most conditions. Overall, across all three estimators, CI coverage is worse under conditions where the point estimator is biased, and coverage rates worsen for meta-analyses with a large number 

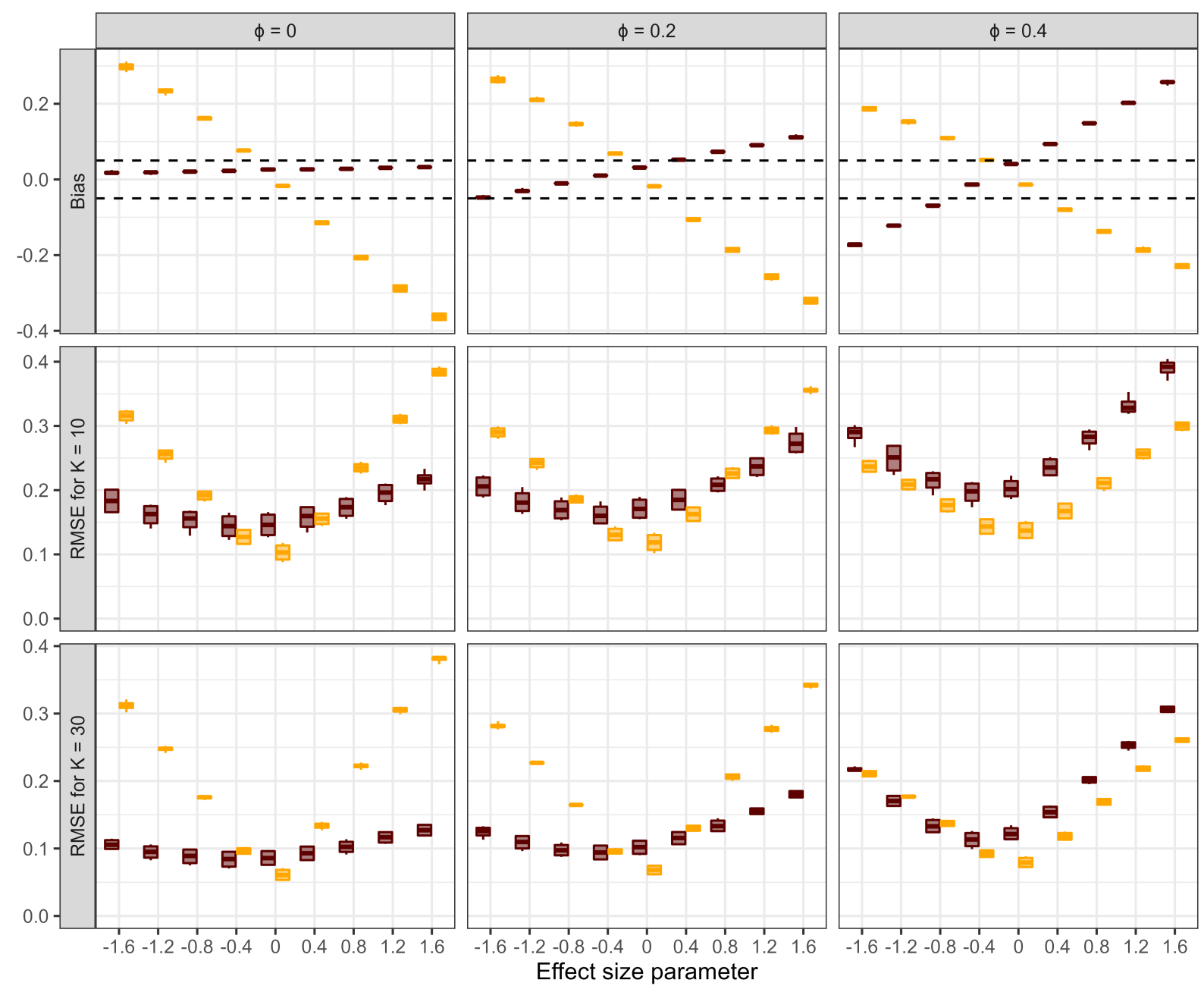

官 OLS

Figure 9. Bias and RMSE of the average WC-SMD estimates.

of studies.

\section{Tau}

Figure 11 illustrates bias and RMSE of the OLS and MLMA estimator for the average Tau across different true effect size values (the horizontal axis) and different auto-correlation values (columns).

Bias. As shown in the first row of Figure 11, the OLS estimator for average Tau is unbiased across the full range of parameter values, even in the presence of autocorrelation. In contrast, the MLMA estimator for average Tau is generally biased for non-null values, and this bias exists across levels of auto-correlation. Because the OLS estimator is unbiased, the bias of MLMA must arise because of the association between the effect size estimates and the weights used in MLMA.

Accuracy. In Figure 11, it can be seen that the OLS estimator is more accurate than the MLMA estimator across all conditions. The accuracy of both estimators appears to be stable across varying degrees of autocorrelation. Overall, both estimators tend to be more accurate for $K=30$ than $K=10$ under most conditions.

Confidence interval coverage. Figure 12 demonstrates that OLS-RVE outperform MLMA or MLMARVE in terms of CI coverage for average Tau. The CI coverage rates of OLS-RVE estimator are close to nominal for most conditions, although they degrade when the true average effect is more extreme (i.e., $\gamma=-0.8$ or 0.8 ). Because of the bias of the MLMA estimator, the CI coverage rates of MLMA and MLMA-RVE are far below nominal for most conditions, and coverage is worse 

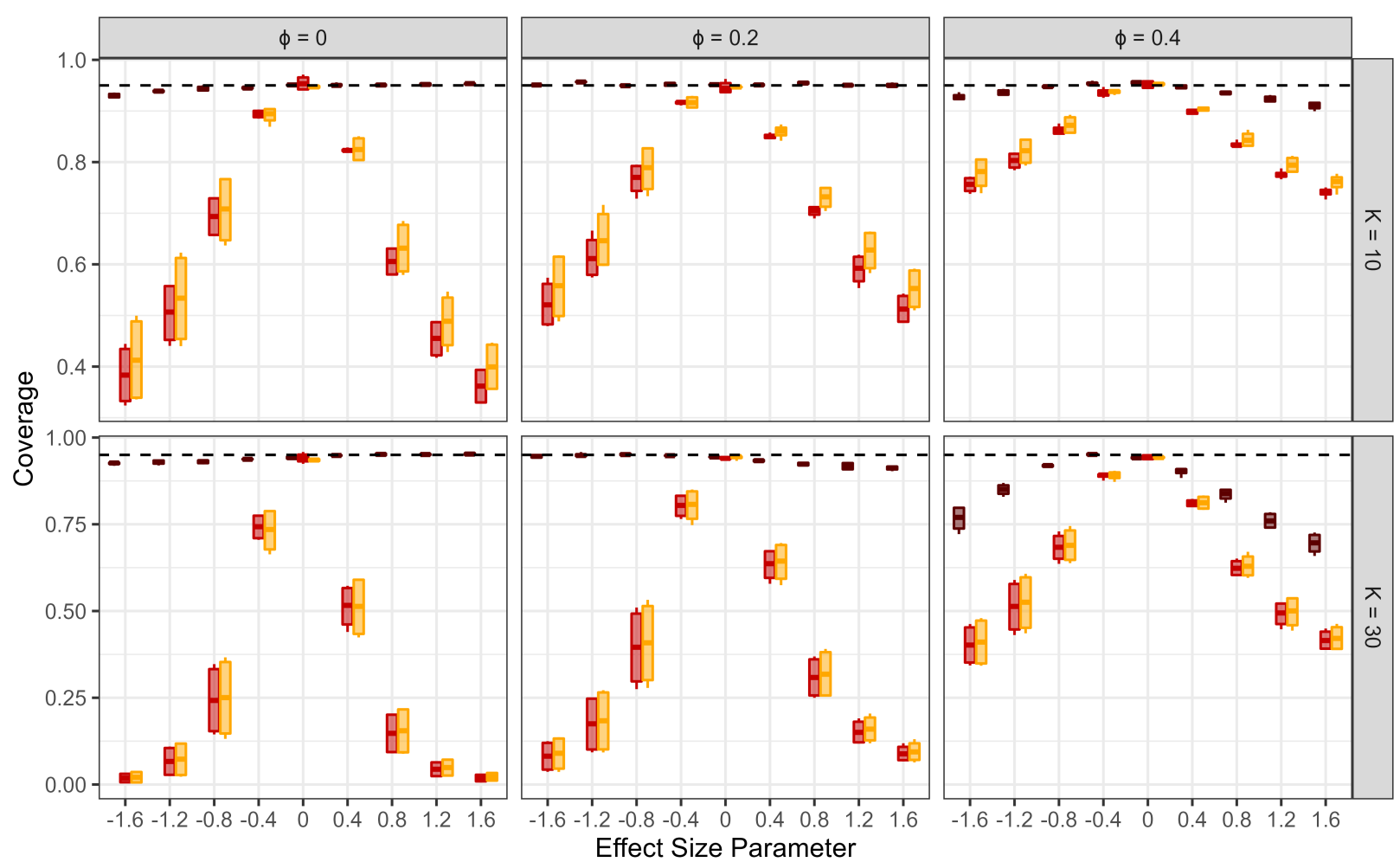

OLS-RVE 官 MLMA

MLMA-RVE

Figure 10. Coverage rate of the confidence intervals for WC-SMD.

when the number of studies is large.

\section{Discussion}

In this study, we used Monte Carlo simulations to examine the performance of several methods of estimating an overall average effect size in meta-analysis of withincase effect sizes from SCEDs. We sought to design a data-generating process that captured many features of real SCED studies, and we investigated estimator performance for three distinct effect size metrics-LRR, WC-SMD, and Tau-in terms of bias, RMSE, and CI coverage rates, under conditions with and without autocorrelation in the raw data series. The supplementary materials include additional findings regarding Type I error rates (Section S3.2) and estimation of the variance components $\left(\tau^{2}\right.$ and $\left.\omega^{2}\right)$ of the MLMA model (Section S3.4).

Many of the core texts on meta-analysis present methods in terms of a generic effect size index, stressing the "conceptual unity of statistical methods" for synthesis of most commonly used indices (Hedges, 2009, p. 25). This principle hinges on the assumption that effect sizes estimators are approximately normally distributed with readily estimated sampling variances. Findings from the present simulation strongly indicate that the conceptual unity principle does not extend to meta-analysis of within-case effect sizes from SCEDs. For the withincase effect size metrics considered here, the short data series typically available in SCEDs are not adequate to ignore the distinct properties of the effect size estimators. Rather, the properties of the effect size estimators and associated sampling variances require consideration when selecting a synthesis approach. We therefore discuss the main findings from the simulations for each effect size metric in turn.

\section{LRR}

For the LRR metric, the OLS and MLMA estimation methods perform differently for average $L R R_{1}$ and average $L R R_{2}$ estimates. First, the MLMA estimator for the average $L R R_{1}$ estimates is approximately unbiased and performs better than OLS estimator in terms of bias and accuracy. In addition, MLMA-RVE has better control of Type I error rates and higher CI coverage rates than MLMA model with conventional standard errors. Accordingly, MLMA-RVE can be used for synthesiz- 

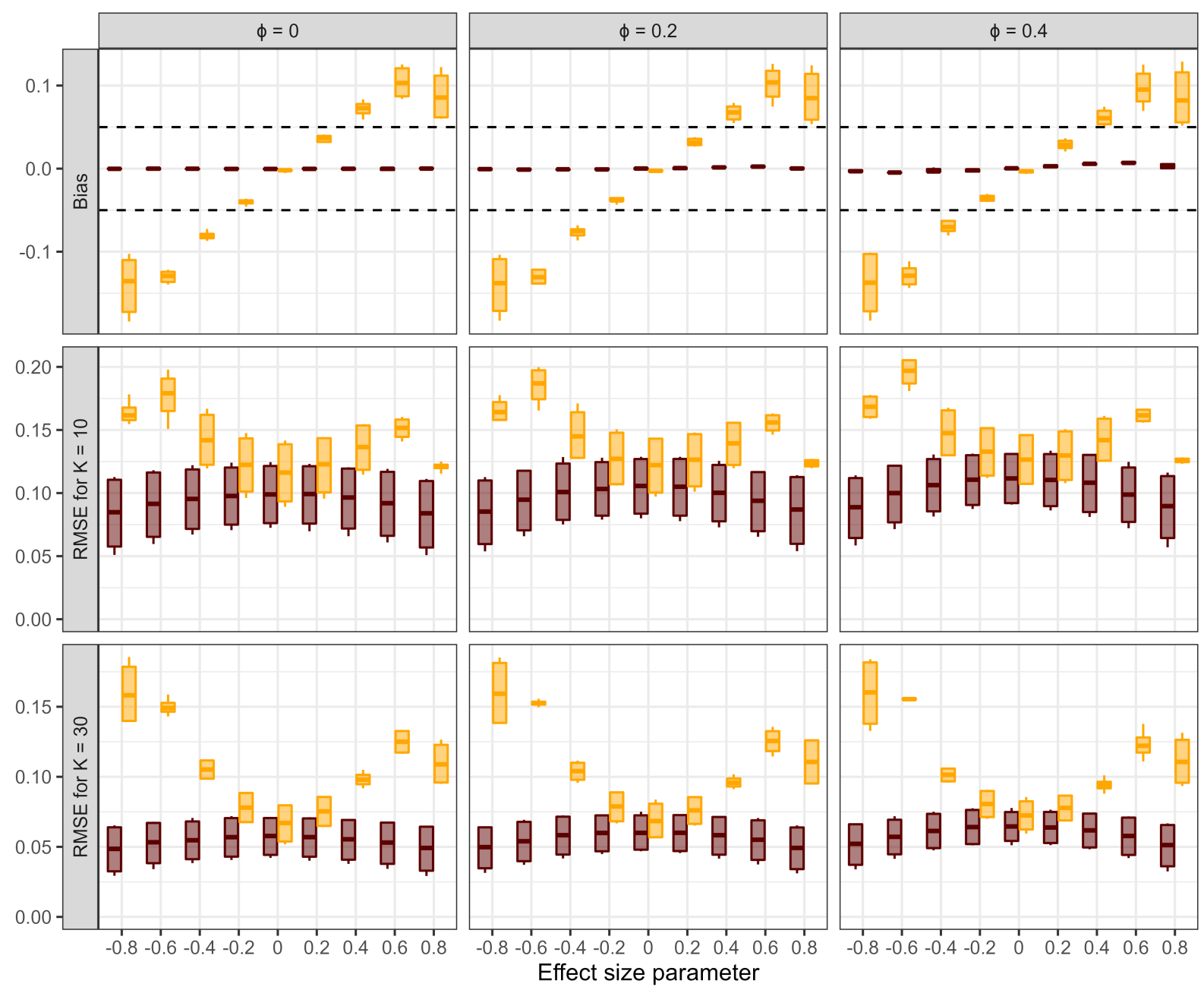

MLMA

Figure 11. Bias and RMSE of the average Tau estimates.

ing $L R R_{1}$ estimates. Second, in the absence of autocorrelation, the OLS estimator for the average $L R R_{2}$ is close to unbiased and performs better than MLMA estimator in terms of bias. In contrast, the MLMA estimator is biased due to the correlation between $L R R_{2}$ estimator and its sampling variance. In the presence of strong auto-correlation, the OLS and MLMA estimator perform similarly in terms of bias, with the latter being more accurate. Both OLS-RVE and MLMA-RVE have near-nominal Type I error rates and OLS-RVE performs similarly to MLMA-RVE in terms of CI coverage rates for most conditions. These patterns suggest that OLSRVE might be used for synthesizing $L R R_{2}$ effect sizes, or used in combination with MLMA-RVE. Finally, under the MLMA model, the $L R R_{1}$ estimator tends to be less biased and more accurate than $L R R_{2}$ estimator (see the Supplementary Section S3.3.2). The CI coverage rates estimated with MLMA-RVE for $L R R_{1}$ are higher than those for $L R R_{2}$. Thus, we recommend using the $L R R_{1}$ effect size estimator with MLMA-RVE for synthesis on the LRR metric.

\section{WC-SMD}

For the WC-SMD metric, neither the OLS nor the MLMA approach performs adequately for meta-analysis when the raw data follow a Poisson AR(1) processeven when used in conjunction with RVE. When autocorrelation is present, both the OLS and MLMA estimators tend to be biased because the case-level WC-SMD effect size estimator is biased. The MLMA estimator has additional problems because the estimated sampling variances of the within-case $g$ are strongly correlated with the effect size estimates themselves, violating one of 


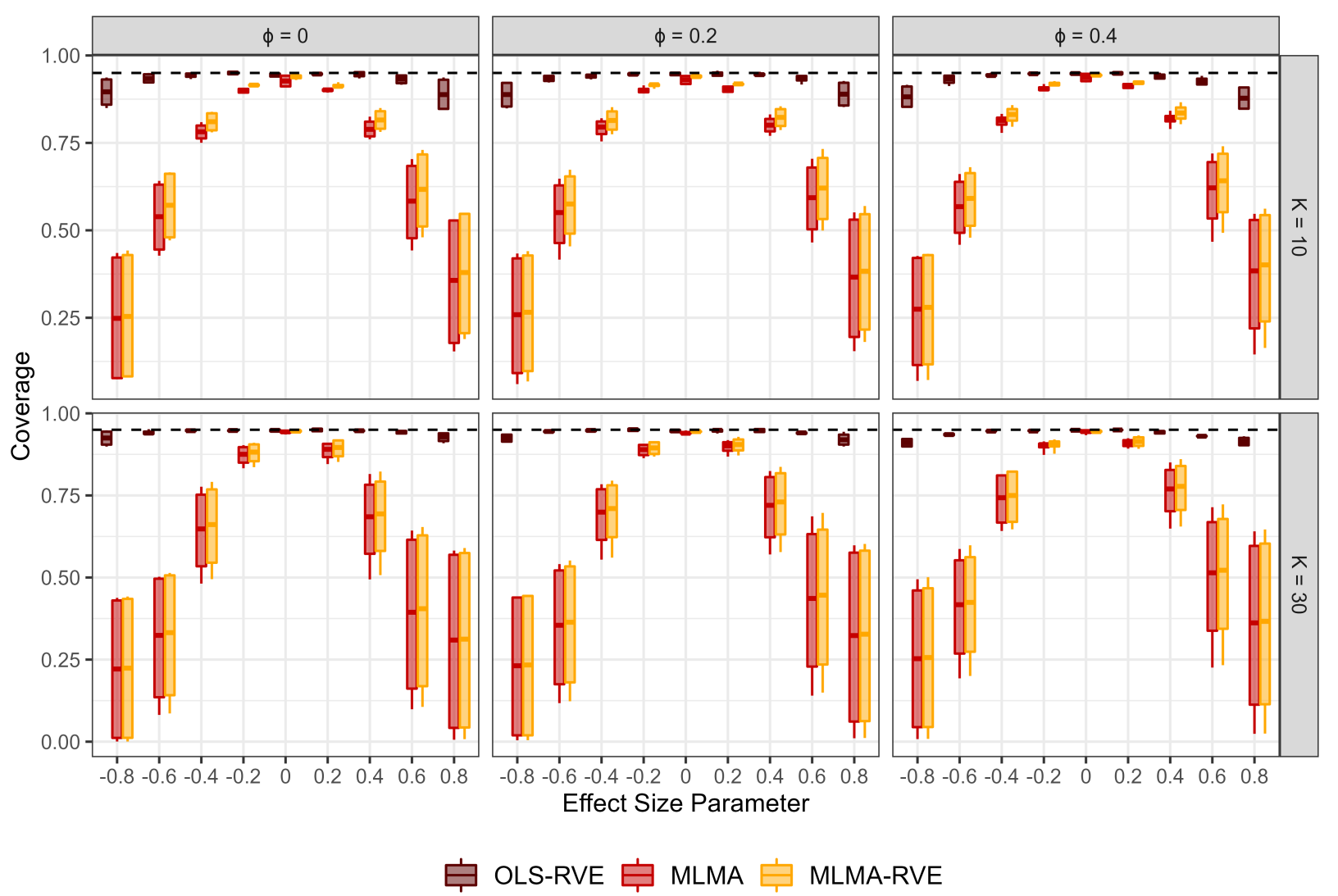

Figure 12. Coverage rate of the confidence intervals for Tau.

the MLMA model assumptions. Moreover, the CI coverage rates for average WC-SMD estimated with MLMA or MLMA-RVE are far below the nominal level, indicating inappropriate statistical inferences about the overall average effects.

In the context of meta-analysis of SMDs from betweengroup designs, Lin \& Aloe (2021) identifed similar problems as those observed here and considered some strategies for remediating bias by using modified estimators of the sampling variance. Such strategies could be considered in further methodological research. Future research should also seek WC-SMD estimators that are less biased by auto-correlation. One candidate is the generalized least squares approach described by Maggin, Swaminathan, et al. (2011), which incorporates auto-correlation into the model for each data series.

In a simulation study examining the performance of onestage raw data synthesis, Petit-Bois et al. (2016) found that the average intervention effects and trend effects are approximately unbiased when the residuals follow firstorder auto-regressive process. This finding might appear to be at odds with our findings regarding the WC-SMD. However, Petit-Bois et al. (2016) studied a model where the fixed effects are un-standardized and the data were generated with Gaussian errors. The finding that the WC-SMD estimator is biased under auto-correlation suggests that raw-data synthesis might encounter problems when based on raw data that has been standardized for each case, as would need to be done for one-stage metaanalysis if studies used different outcome measures. In further research, it would be useful to include head-tohead comparisons between raw-data synthesis and synthesis of within-case effect size estimators and to find methods of estimating scale parameters that are less biased by auto-correlation.

\section{Tau}

For synthesizing Tau effect sizes, the OLS estimator substantially out-performs the MLMA estimator. The OLS estimator remains unbiased because the Tau effect size estimator is essentially unbiased, even in the presence of auto-correlation, and OLS-RVE produces CIs with closeto-nominal coverage rates. In contrast, the MLMA estimator is noticeably biased, for several possible reasons. One possible cause is that the effect size estimator is correlated with its variance estimator and the sampling 
variance strongly depends on the true effect size, properties that violate the assumptions of the MLMA model. Another possible cause is that the distributional assumptions of the MLMA are not adequate approximations for the true data-generating process that we examined, which involved using shifted-and-scaled beta distributions. According to these findings, OLS-RVE might be used in estimating the average Tau effect size in meta-analysis of SCEDs. Because Tau is a linear transformation of NAP, results of Tau also apply to NAP.

Tau differs from the LRR and WC-SMD metrics in that its range is limited to the interval -1 to 1 . This restriction created several challenges for simulating raw data consistent with the MLMA model in the Tau metric, such as needing to use shifted-and-scaled beta distributions to generate study-level and case-level effect size parameters. One possible alternative approach for synthesizing Tau would be to use a meta-analytic model for a transformation of the Tau parameter, such as a Fisher $z$ transformation or, equivalently, a logistic transformation of the NAP parameter. Ryu \& Agresti (2008) proposed methods of analyzing an effect size measure equivalent to NAP for measuring difference between two ordinal variables. They discussed the use of a logit model for estimating the proposed ordinal effect size across different predictor variables - an approach akin to a fixed effect meta-regression. In might be possible to extend this approach to include random effects for multiple effect sizes from multiple studies. Such extensions could provide improvements over the linear MLMA with Gaussian errors, as we have investigated here.

\section{Limitations and suggestions for future research}

This study has several limitations that are important to note. First, in the meta-analysis simulations, we generated raw data using a Poisson AR(1) model for frequency counts outcomes. This is a fairly natural model for count outcomes, which are prevalent in SCEDs, yet it is also a best-case scenario for use of LRR effect sizes. In Supplementary Section S2, we examined the behavior of the effect size estimators and variance estimators under a Gaussian AR(1) model. The similarity of findings with the Poisson AR(1) model does suggest that the meta-analytic estimators would perform similarly with normally distributed (Gaussian) outcomes. However, the patterns of performance we have described might not generalize to all other types of outcomes.

Second, the simulation methods in this paper might not reflect the complex meta-analytic context with a mixture of different designs. For example, we generated data series where the phase lengths in the baseline and intervention conditions were determined through a random process, yielding a data structure similar to a multiplebaseline design with randomly selected start points. However, SCEDs often use a response-guided process to decide when to start the intervention (Ferron, Joo, \& Levin, 2017). Swan, Pustejovsky, \& Beretvas (2020) demonstrated some plausible response-guided processes cause downward bias in estimates of baseline variability, which is more severe when auto-correlation is present. As a consequence, there might be more bias in effect size estimators that depend on the estimation of baseline variability, such as the WC-SMD, which could lead in turn to stronger bias in average effect sizes and inappropriate inferences in meta-analysis. Thus, further investigation is needed to examine the implications of response-guide designs in the meta-analysis context.

Third, we used a limited set of parameter values for the simulation. There is relatively little empirical evidence available regarding typical levels of between-study and within-study heterogeneity. More research is needed for investigating performance across a wider range of heterogeneity levels, as well as across a broader range of auto-correlation.

Fourth, the three effect size metrics investigated in this study are limited to be used for single-case design data that are stable without trends. Given that some singlecase data could demonstrate systematic time trends that might affect the implications of auto-correlation, future research could investigate the performance of those estimators when time trends are ignored. Researchers could also examine extensions of the effect size estimators based on models that handle linear time trends or nonlinear time trends (Maggin, Swaminathan, et al., 2011; e.g., Swan \& Pustejovsky, 2018; Tarlow, 2017). Until such extensions are available, researchers conducting metaanalyses of SCED data using the LRR, WC-SMD, or Tau metric should carefully consider whether it is reasonable to assume stability within phases.

Finally, we simulated univariate outcome per case. As shown in some applied meta-analytic research (Collins et al., 2020; Ledford \& Pustejovsky, 2020), SCEDs often include multiple outcomes assessed on some or all cases. Consequently, it would be useful to consider how the methods examined here could be extended to multivariate meta-analysis of SCEDs.

\section{Conclusions}

Notwithstanding the limitations we have noted, findings from this simulation have several implications for how to approach meta-analysis of single-case experimental designs using LRR, WC-SMD, or Tau. First, the MLMA model works properly for synthesis of $L R R_{1}$ estimators. 
Applying RVE to MLMA model provides better control of Type I error rates and higher CI coverage rates and thus is recommended. Thus, we would recommend researchers use MLMA in conjunction with RVE in the meta-analysis of $L R R_{1}$ effect sizes for frequency count outcomes. Second, although RVE can handle the inaccurate sampling variance estimates due to auto-correlation, it cannot correct for systematic bias in effect size estimates. Therefore, we would not recommend researchers use either OLS or MLMA approach for meta-analyzing WC-SMD effect sizes for frequency count outcomes. If the outcomes are on the same scale across studies, researchers could consider using raw data meta-analytic model (Petit-Bois et al., 2016) to obtain an unbiased overall average effect size. Third, meta-analysts who are interested in estimating the average Tau or NAP effect size should consider using OLS-RVE rather than MLMA. We emphasize that these recommendations are, however, limited to the contexts where the effect sizes are appropriate summary metrics for the data from included designs (i.e., they would not apply to collections of studies where the data series exhibit systematic time trends).

Although the MLMA model has already been applied in syntheses of SCEDs, no methodological research has examined whether it works well for different effect size metrics. Results from this simulation study suggest that MLMA model performs properly for some effect size measures but not for others. Further methodological development on synthesis and meta-analysis of SCEDs needs to pay attention to the statistical properties of different effect size metrics and to evaluate the meta-analytic methods in simulations across multiple effect size measures. By developing simulation models that capture the important features of real data series, methodological research will be able to provide better guidance about how to synthesize results from SCEDs.

\section{References}

Acion, L., Peterson, J. J., Temple, S., \& Arndt, S. (2006). Probabilistic index: An intuitive non-parametric approach to measuring the size of treatment effects. Statistics in Medicine, 25(4), 591-602. https://doi. org/10.1002/sim.2256

Anderson, T. W. (1994). The statistical analysis of time series. John Wiley \& Sons.

Barnard-Brak, L., Watkins, L., \& Richman, D. M. (2021). Autocorrelation and estimates of treatment effect size for single-case experimental design data. Behavioral Interventions.

Bene, K., Banda, D. R., \& Brown, D. (2014). A metaanalysis of peer-mediated instructional arrangements and autism. Review Journal of Autism and Developmental Disorders, 1(2), 135-142.

Borenstein, M. (2009). Effect sizes for continuous data. In H. M. Cooper, L. V. Hedges, \& J. C. Valentine (Eds.), The handbook of research synthesis and metaanalysis (pp. 221-236). New York, NY: Russell Sage Foundation.

Busk, P. L., \& Serlin, R. C. (1992). Meta-analysis for single-case research. In T. R. Kratochwill \& J. R. Levin (Eds.), Single-case research design and analysis: New directions for psychology and education (pp. 187-212). Hillsdale, NJ: Lawrence Erlbaum Associates, Inc.

Chen, M., \& Pustejovsky, J. E. (2021a). Multi-level meta-analysis of single-case experimental designs using robust variance estimation. https://doi.org/10. 31234/osf.io/59h32

Chen, M., \& Pustejovsky, J. E. (2021b). Supplementary materials [code and complete numerical simulation results] to: Multi-level meta-analysis of single-case experimental designs using robust variance estimation. Retrieved from https://osf.io/zypsh

Collins, T. A., Drevon, D. D., Brown, A. M., Villarreal, J. N., Newman, C. L., \& Endres, B. (2020). Say something nice: A meta-analytic review of peer reporting interventions. Journal of School Psychology, 83, 89103.

Cook, B., Buysse, V., Klingner, J., Landrum, T., McWilliam, R., Tankersley, M., \& Test, D. (2014). Council for exceptional children: Standards for evidence-based practices in special education. Teaching Exceptional Children, 46(6), 206.

Declercq, L., Jamshidi, L., Fernandez Castilla, B., Moeyaert, M., Beretvas, S. N., Ferron, J. M., \& Van den Noortgate, W. (2020). Multilevel meta-analysis of individual participant data of single-case experimental designs: One-stage versus two-stage methods. Multivariate Behavioral Research, 1-20.

Ferron, J. M., Joo, S.-H., \& Levin, J. R. (2017). A monte carlo evaluation of masked visual analysis in response-guided versus fixed-criteria multiplebaseline designs. Journal of Applied Behavior Analysis, 50(4), 701-716.

Froján-Parga, M. X., Prado-Gordillo, M. N. de, ÁlvarezIglesias, A., \& Alonso-Vega, J. (2019). Functional behavioral assessment-based interventions on adults' delusions, hallucinations and disorganized speech: A single case meta-analysis. Behaviour Research and Therapy, 120, 103444.

Gingerich, W. J. (1984). Meta-analysis of applied time-series data. Journal of Applied Behavioral Science, 20(1), 71-79. https://doi.org/10.1177/ 002188638402000113

Hedges, L. V. (1981). Distribution theory for Glass's es- 
timator of effect size and related estimators. Journal of Educational Statistics, 6(2), 107-128. https: //doi.org/10.3102/10769986006002107

Hedges, L. V. (2009). Statistical considerations. In H. M. Cooper, L. V. Hedges, \& J. C. Valentine (Eds.), The handbook of research synthesis and meta-analysis (pp. 38-47). New York, NY: Russell Sage Foundation.

Hedges, L. V., Tipton, E., \& Johnson, M. C. (2010). Robust variance estimation in meta-regression with dependent effect size estimates. Research Synthesis Methods, 1(1), 39-65.

Horner, R. H., \& Odom, S. L. (2014). Constructing single-case research designs: Logic and options. In T. R. Kratochwill \& J. R. Levin (Eds.), Single-case intervention research: Methodological and statistical advances (pp. 27-51). Washington, DC: American Psychological Association.

Horner, R. H., Swaminathan, H., Sugai, G., \& Smolkowski, K. (2012). Considerations for the systematic analysis and use of single-case research. $E d$ ucation and Treatment of Children, 35(2), 269-290. https://doi.org/10.1353/etc.2012.0011

Jamshidi, L., Heyvaert, M., Declercq, L., FernándezCastilla, B., Ferron, J., Moeyaert, M., \& Van den Noortgate, W. (2020). A systematic review of singlecase experimental design meta-analyses: Characteristics of study designs, data, and analyses. EvidenceBased Communication Assessment and Intervention.

Kaiser, A. P. (2014). Using single case designs in comprehensive programs of research. In T. R. Kratochwill \& J. R. Levin (Eds.), Single-case intervention research:methodological and statistical advances (pp. 309-323). Washington, DC: American Psychology Association.

Konstantopoulos, S. (2011). Fixed effects and variance components estimation in three-level meta-analysis. Research Synthesis Methods, 2(1), 61-76.

Kratochwill, T. R., \& Levin, J. R. (2014). Enhancing the scientific credibility of single-case intervention research: Randomization to the rescue. In $\mathrm{T}$. R. Kratochwill \& J. R. Levin (Eds.), Single-case intervention research: Methodological and statistical advances (pp. 53-89). Washington, DC: American Psychological Association.

Ledford, J. R., \& Pustejovsky, J. E. (2020). Systematic review and meta-analysis of stay-play-talk interventions for improving social behaviors of young children. Journal of Positive Behavior Interventions, 1098300720983521.

Lin, L., \& Aloe, A. M. (2021). Evaluation of various estimators for standardized mean difference in metaanalysis. Statistics in Medicine, 40(2), 403-426.

Maggin, D. M., O’Keeffe, B. V., \& Johnson, A. H.
(2011). A quantitative synthesis of methodology in the meta-analysis of single-subject research for students with disabilities: 1985-2009. Exceptionality, 19(2), 109-135. https://doi.org/10.1080/09362835. 2011.565725

Maggin, D. M., Pustejovsky, J. E., \& Johnson, A. H. (2017). A meta-analysis of school-based group contingency interventions for students with challenging behavior: An update. Remedial and Special Education, 38(6), 353-370.

Maggin, D. M., Swaminathan, H., Rogers, H. J., O'keeffe, B. V., Sugai, G., \& Horner, R. H. (2011). A generalized least squares regression approach for computing effect sizes in single-case research: Application examples. Journal of School Psychology, 49(3), 301-321.

Manolov, R., \& Moeyaert, M. (2017). How Can SingleCase Data Be Analyzed? Software Resources, Tutorial, and Reflections on Analysis. Behavior Modification, 41(2), 179-228. https://doi.org/10.1177/ 0145445516664307

Matyas, T. A., \& Greenwood, K. M. (1996). Serial dependency in single-case time series. In R. D. Franklin, D. B. Allison, \& B. S. Gorman (Eds.), Design and Analysis of Single-Case Research (pp. 215243). Mahwah, NJ: Lawrence Erlbaum.

McKenzie, E. (1988). Some ARMA models for dependent sequences of Poisson counts. Advances in Applied Probability, 20(4), 822-835. https://doi.org/10. 2307/1427362

Mee, R. W. (1990). Confidence Intervals for Probabilities and Tolerance Regions Based on a Generalization of the Mann-Whitney Statistic. Journal of the American Statistical Association, 85(411), 793. https://doi.org/10.2307/2290017

Moeyaert, M., Ugille, M., Ferron, J. M., Beretvas, S. N., \& Van den Noortgate, W. (2013). The threelevel synthesis of standardized single-subject experimental data: A monte carlo simulation study. Multivariate Behavioral Research, 48(5), 719-748. https: //doi.org/10.1080/00273171.2013.816621

Parker, R. I., \& Vannest, K. J. (2009). An improved effect size for single-case research: Nonoverlap of all pairs. Behavior Therapy, 40(4), 357-367. https: //doi.org/10.1016/j.beth.2008.10.006

Parker, R. I., Vannest, K. J., \& Davis, J. L. (2011). Effect Size in Single-Case Research: A Review of Nine Nonoverlap Techniques. Behavior Modification, 35(4), 303-322. https://doi.org/10.1177/ 0145445511399147

Parker, R. I., Vannest, K. J., Davis, J. L., \& Sauber, S. B. (2011). Combining nonoverlap and trend for singlecase research: Tau-u. Behavior Therapy, 42(2), 284299. 
Petit-Bois, M., Baek, E. K., Van den Noortgate, W., Beretvas, S. N., \& Ferron, J. M. (2016). The consequences of modeling autocorrelation when synthesizing single-case studies using a three-level model. Behavior Research Methods, 48(2), 803-812.

Pustejovsky, J. E. (2015). Measurement-comparable effect sizes for single-case studies of free-operant behavior. Psychological Methods, 20(3), 342-359. https://doi.org/10.1037/met0000019

Pustejovsky, J. E. (2018). Using response ratios for meta-analyzing single-case designs with behavioral outcomes. Journal of School Psychology, 68, 99112.

Pustejovsky, J. E. (2019). Procedural sensitivities of effect sizes for single-case designs with directly observed behavioral outcome measures. Psychological Methods, 24(2), 217.

Pustejovsky, J. E. (2020). clubSandwich: Clusterrobust (sandwich) variance estimators with smallsample corrections. Retrieved from https://github. com/jepusto/clubSandwich

Pustejovsky, J. E., \& Ferron, J. M. (2017). Research synthesis and meta-analysis of single-case designs. In J. M. Kauffman, D. P. Hallahan, \& P. C. Pullen (Eds.), Handbook of special education (pp. 168-186). Routledge New York, NY.

Pustejovsky, J. E., Swan, D. M., \& English, K. W. (2019). An examination of measurement procedures and characteristics of baseline outcome data in single-case research. Behavior Modification, 0145445519864264.

R Core Team. (2020). R: A language and environment for statistical computing. Vienna, Austria: R Foundation for Statistical Computing. Retrieved from https://www.R-project.org/

Randolph, J. J. (2007). Meta-analysis of the research on response cards: Effects on test achievement, quiz achievement, participation, and off-task behavior. Journal of Positive Behavior Interventions, 9(2), 113-128.

Ryu, E., \& Agresti, A. (2008). Modeling and inference for an ordinal effect size measure. Statistics in Medicine, 27(10), 1703-1717.

Sen, P. K. (1967). A note on asymptotically distributionfree confidence bounds for $\mathrm{p}\{\mathrm{x}<\mathrm{y}\}$, based on two independent samples. Sankhyā: The Indian Journal of Statistics, Series A, 95-102.

Shadish, W. R., Hedges, L. V., \& Pustejovsky, J. E. (2014). Analysis and meta-analysis of single-case designs with a standardized mean difference statistic: A primer and applications. Journal of School Psychology, 52(2), 123-147. https://doi.org/10.1016/j. jsp.2013.11.005

Shadish, W. R., \& Sullivan, K. J. (2011). Characteris- tics of single-case designs used to assess intervention effects in 2008. Behavior Research Methods, 43(4), 971-980.

Solomon, B. G. (2014). Violations of assumptions in school-based single-case data: Implications for the selection and interpretation of effect sizes. Behavior Modification, 38(4), 477-496.

Swan, D. M., \& Pustejovsky, J. E. (2018). A gradual effects model for single-case designs. Multivariate Behavioral Research, 53(4), 574-593.

Swan, D. M., Pustejovsky, J. E., \& Beretvas, S. N. (2020). The impact of response-guided designs on count outcomes in single-case experimental design baselines. Evidence-Based Communication Assessment and Intervention, 14(1-2), 82-107.

Tarlow, K. R. (2017). An improved rank correlation effect size statistic for single-case designs: Baseline corrected Tau. Behavior Modification, 41(4), 427467. https://doi.org/10.1177/0145445516676750

Tipton, E. (2015). Small sample adjustments for robust variance estimation with meta-regression. Psychological Methods, 20(3), 375.

Ugille, M., Moeyaert, M., Beretvas, S. N., Ferron, J. M., \& Van den Noortgate, W. (2014). Bias corrections for standardized effect size estimates used with single-subject experimental designs. The Journal of Experimental Education, 82(3), 358-374. https: //doi.org/10.1080/00220973.2013.813366

Van den Noortgate, W., \& Onghena, P. (2008). A multilevel meta-analysis of single-subject experimental design studies. Evidence-Based Communication Assessment and Intervention, 2(3), 142-151. https: //doi.org/10.1080/17489530802505362

Viechtbauer, W. (2010). Conducting meta-analyses in $\mathrm{R}$ with the metafor package. Journal of Statistical Software, 36(3), 1-48. Retrieved from https: //www.jstatsoft.org/v36/i03/

Wahman, C. L., Pustejovsky, J. E., Ostrosky, M. M., \& Santos, R. M. (2019). Examining the effects of social stories ${ }^{\mathrm{TM}}$ on challenging behavior and prosocial skills in young children: A systematic review and meta-analysis. Topics in Early Childhood Special Education, 0271121419855692.

What Works Clearinghouse. (2020). WWC procedures and standards handbook (version 4.1). Washington, DC: US department of education, institute of education sciences. National Center for Education Evaluation and Regional Assistance, What Works Clearinghouse.

Wolery, M., Busick, M., Reichow, B., \& Barton, E. E. (2010). Comparison of overlap methods for quantitatively synthesizing single-subject data. The Journal of Special Education, 44(1), 18-28.

Zimmerman, K. N., Pustejovsky, J. E., Ledford, J. R., 
Barton, E. E., Severini, K. E., \& Lloyd, B. P. (2018). Single-case synthesis tools II: Comparing quantitative outcome measures. Research in Developmental Disabilities, 79, 65-76.

\section{Appendix}

In order to generate raw data based on an AR(1) Poisson model, we needed to determine the mean level of the intervention phase $\left(\mu_{B}\right)$ given the mean baseline level $\mu_{A}$ and an effect size parameter. Here we explain how to determine $\mu_{B}$ for each effect size metric that we examined.

LRR. The log response ratio effect size parameter is defined as $\lambda=\ln \left(\mu_{B} / \mu_{A}\right)$. Given $\mu_{A}$ and $\lambda, \mu_{B}$ can be calculated as $\mu_{B}=\mu_{A} \times \exp (\lambda)$, where $\exp ()$ denotes the exponential function.

WC-SMD. The WC-SMD effect size parameter is defined as $\delta=\left(\mu_{B}-\mu_{A}\right) / \sigma_{A}$, where and $\sigma_{A}$ is the standard deviation of the baseline phase outcome distribution. Under the AR(1) Poisson model, outcomes in the baseline phase are marginally Poisson-distributed with mean $\mu_{A}$ and variance $\sigma_{A}^{2}=\mu_{A}$. Thus, given $\mu_{A}$ and $\delta, \mu_{B}$ can be calculated as $\mu_{B}=\mu_{A}+\delta \times \sqrt{\mu_{A}}$.

Tau. For an outcome where decrease is desirable, the Tau effect size parameter for a given case is defined as

$$
\theta=2 \times \operatorname{Pr}\left(Y_{A}>Y_{B}\right)+\operatorname{Pr}\left(Y_{A}=Y_{B}\right)-1 .
$$

Under the assumptions of the AR(1) Poisson model, $Y^{A} \sim \operatorname{Poisson}\left(\mu_{A}\right)$ and $Y^{B} \sim \operatorname{Poisson}\left(\mu_{B}\right)$. Assuming that outcome series are mutually independent, the random variable $S=Y^{A}-Y^{B}$ follows a Skellam distribution such that $S \sim \operatorname{Skellam}\left(\mu_{A}, \mu_{B}\right)$. Thus, the Tau parameter for one data series is equal to

$$
\begin{aligned}
\theta & =\operatorname{Pr}(S=0)+2 \times \operatorname{Pr}(S<0)-1 \\
& =f_{S}\left(0 \mid \mu_{A}, \mu_{B}\right)+1-2 \times F_{S}\left(0 \mid \mu_{A}, \mu_{B}\right),
\end{aligned}
$$

where $f_{S}$ is the probability mass function and $F_{S}$ is the cumulative distribution function of the Skellam distribution. Thus, given $\mu_{A}$ and the simulated $\theta, \mu_{B}$ can calculated by solving Equation (22).

When $Y^{A}$ and $Y^{B}$ are Poisson-distributed, the range of $\tau$ is restricted based on $\mu_{A}$. Given $\mu_{A}>0$, the maximum possible value of $\theta$ occurs when $\mu_{B}=0$. Therefore, $\theta$ must be less than or equal to $\theta_{\max }=f_{S}\left(0 \mid \mu_{A}, 0\right)+1-2 \times$ $F_{S}\left(0 \mid \mu_{A}, 0\right)$. For $\mu_{A}=1,2,3,4$, or 5 , this bound is equal to $0.632,0.865,0.950,0.982$, or 0.993 , respectively. 